REVIEW

\title{
Effect of oral contraceptives and hormone replacement therapy on bone mineral density in premenopausal and perimenopausal women: a systematic review
}

\author{
S L Liv, C M Lebrun
}

Br J Sports Med 2006;40:1 1-24. doi: 10.1136/bjsm.2005.020065

Seventy five articles on the effect of oral contraceptives and other hormone replacement on bone density in premenopausal and perimenopausal women were reviewed. The evidence was appraised using the Oxford Centre for Evidence-Based Medicine levels of evidence. There is good evidence for a positive effect of oral contraceptives on bone density in perimenopausal women, and fair evidence for a positive effect in "hypothalamic" oligo/amenorrhoeic premenopausal women. There is limited evidence for a positive effect in healthy and anorexic premenopausal women. In hypothalamic oligo/ amenorrhoeic women, baseline bone density has been shown to be significantly lower than that in healthy controls, therefore the decision to treat is clinically more important. The ideal formulation(s) and duration of treatment remain to be determined by further longitudinal and prospective randomised controlled trials in larger subject populations.

See end of article for authors' affiliations

Correspondence to: Dr Lebrun, Fowler Kennedy Sports Medicine Clinic, University of Western Ontario, London, Ontario N6A 3K7, Canada;

clebrun@uwo.ca

Accepted

5 September 2005
A ccording to Statistics Canada's 1996-1997 National Health Population Survey, 18\% of Canadian women aged 15-49 use oral contraceptives (OCs). ${ }^{1}$ In female athletes, OC use is at least as common as in the general population. ${ }^{2}$ The health benefits of OCs are contraceptive-for example, pregnancy prevention, reduced risk of ectopic pregnancy-and non-contraceptive-for example, cycle control, prevention of ovarian cancer, and reduction in dysmenorrhoea and acne. ${ }^{3}$ Whereas the pharmacological effects of both oestrogen and progesterone on bone metabolism are widely supported in the literature, the clinical effects of OC use on bone mineral density (BMD) remain unclear. Conflicting views may stem from the many confounding variables that affect BMD, including age, race, genetics, illness, smoking, weight, exercise, diet, and oestrogen status. ${ }^{4}$ The last four are especially relevant to the female athlete population, in light of the increasing prevalence of the female athlete triad. Compared with the general population, the higher levels of impact loading (in the setting of inadequate hormonal and nutritional status) may increase the female athlete's risk of fractures and other skeletal injuries. Consequently, the female athlete faces unique concerns with respect to bone health; thus any effects of sustained OC use on BMD are of paramount importance. This review critically examines the literature to determine the effect of OCs and other forms of hormone therapy on BMD in four groups of women: healthy premenopausal, "hypothalamic" oligo/ amenorrhoeic, anorexic premenopausal, and perimenopausal.

\section{THE FEMALE ATHLETE TRIAD}

First described in the early 1990s, the female athlete triad is a clinical syndrome comprising one or more of three specific components: disordered eating, amenorrhoea, and osteoporosis. $^{5}$ The World Health Organization (WHO) classifies BMD by $\mathrm{T}$ score-that is, the number of standard deviations below peak BMD-as follows: $<-1$ is normal; -1 to -2.5 is osteopenia; $>-2.5$ is osteoporosis. ${ }^{6}$ However, the International Society for Clinical Densitometry claims that the WHO classification should not be applied to healthy premenopausal women because it is based on studies in postmenopausal women. ${ }^{7}$ Further, recent data suggest that the female athlete triad should use osteopenia as a defining criterion rather than osteoporosis, to more accurately reflect the greater prevalence of osteopenia in the female athlete population. ${ }^{8}$

The female athlete triad is characterised by a negative energy balance, created when energy expenditure exceeds intake. This can be due to inadequate energy intake, excessive exercise, or a combination of both. A negative energy balance invariably leads to disruption of the hypothalamic-pituitary-ovarian axis, ovarian suppression, and various forms of menstrual dysfunction (including shortened luteal phase, oligomenorrhoea, and amenorrhoea). Ultimately, hypooestrogenism ${ }^{9}$ and the nutritional deficits contribute to the development of decreased BMD. Management of the female athlete triad is multidisciplinary, involving doctors, psychologists, and nutritionists. However, the use of OCs to treat decreased BMD found in patients with the female athlete triad is controversial.

\section{PHYSIOLOGICAL EFFECTS OF OESTROGEN AND EXERCISE ON BONE}

Oestrogen plays a critical role in skeletal homoeostasis, with well recognised beneficial effects

Abbreviations: BMD, bone mineral density; DXA, dual energy $x$ ray absorptiometry; IGF-I, insulin-like growth factor I; OC, oral contraceptive; RCT, randomised controlled trial 
on bone mass, but the mechanisms by which it acts remain unclear. At the cellular level, oestrogen exerts effects on both osteoclast and osteoblast function, resulting in tonic inhibition of bone turnover and maintenance of the balance between bone resorption and formation. ${ }^{10}$ It is believed that oestrogen acts directly on bone cells in a receptor mediated manner, as suggested by oestrogen receptor expression in both osteoblasts ${ }^{11}$ and osteoclasts. ${ }^{12}$ However, oestrogen also mediates indirect actions on bone through effects on hormones, such as calcitonin and parathyroid hormone, and on cytokines and growth factors. ${ }^{13}$

Exercise also has an important effect on BMD. It has been proposed that bone is capable of sensing biomechanical strain through an internal "mechanostat", and adjusts the level of remodelling accordingly to increase bone accretion. ${ }^{14}$ This pathway is oestrogen dependent, as oestrogen deficiency alters the set point of the mechanostat, thereby impairing detection of biomechanical strain. ${ }^{10}$ The result is an inadequate level of bone remodelling and accretion. Chronically impaired response to strain and persistent inadequate bone remodelling and accretion potentially contribute to bone loss. Therefore, in physically active hypo-oestrogenic womenthat is, women with the female athlete triad-OCs may be beneficial in "resetting" the mechanostat and restoring the appropriate homoeostatic response of bone to exercise.

\section{METHODS}

\section{Study selection}

The electronic databases Medline, the Cochrane database of systematic reviews (CDSR), ACP journal club, database of abstracts of reviews of effects (DARE), Cochrane central register of controlled trials (CCTR), cumulative index to nursing and allied health literature (CINAHL), and SPORTDiscus were searched to identify potentially relevant articles up until March 2005. Searches used a combination of medical subject headings and keywords (table 1).

There were 327 hits from Medline, 212 from CINAHL, 30 from CDSR, ACP journal club, DARE, and CCTR (combined), and 17 from SPORTDiscus. Titles and abstracts were scanned to eliminate duplicates and to assess for relevance. Additional references were found through bibliographic searches of all retrieved articles.

Studies were included if they (a) examined effects on BMD, (b) included healthy, "hypothalamic" oligo/amenorrhoeic, or anorexic premenopausal or perimenopausal women, and (c) included oestrogen and/or progesterone replacement therapy-that is, OCs or hormone replacement therapy—as a treatment.

\section{Quality assessment and data extraction}

The quality of evidence was appraised using the Oxford Centre for Evidence-Based Medicine levels of evidence, ${ }^{15}$ based on study design, including: sample size, randomisation, specific inclusion criteria, adequate follow up, and blinding (table 2).

Articles were classified into one of four groups according to study population (healthy premenopausal, "hypothalamic" oligo/amenorrhoeic premenopausal, anorexic premenopausal, perimenopausal), then subdivided by study design (randomised controlled trial (RCT), cohort, cross sectional, case series, case report) and by effect (positive, negative, no effect). Data summarised include OC exposure (formulation, dose) and outcome (measurement of BMD).

\section{RESULTS}

\section{Study selection}

Seventy five studies were reviewed ${ }^{16-90}$ : 11 RCTs, ${ }^{26-29} 626369$ 74768028 cohort, ${ }^{16-18} 30-38$ 55-58 64-68 $70777981-8432$ cross sectional, ${ }^{19-}$ 25 39-53 59-61 $727385-89$ three case series, ${ }^{54} 7890$ and one case report ${ }^{71}$ (table 3). Tables 4-14 give descriptions of each study. The results focus on RCTs, as they provide the strongest evidence.

\section{Data extraction}

Healthy premenopausal women

Forty six studies in healthy premenopausal women were reviewed. Ten (three cohort, ${ }^{16-18}$ seven cross sectional ${ }^{19-25}$ ) showed a positive effect, 29 (four RCTs, ${ }^{26-29}$ nine cohort, ${ }^{30-38} 15$ cross sectional, ${ }^{39-53}$ one case series ${ }^{54}$ ) showed no effect, and seven (four cohort, ${ }^{55-58}$ three cross sectional ${ }^{59-61}$ ) showed a negative effect. All of the RCTs showed no effect on BMD, as measured by either dual energy $x$ ray absorptiometry $(\mathrm{DXA})^{262729}$ or quantitative computed tomography. ${ }^{28}$ However, three of the four RCTs also showed a positive effect on bone turnover, as shown by decreased urinary concentrations of the bone resorption markers pyridinoline, deoxypyridinoline, ${ }^{27}{ }^{29}$ and cross linked $\mathrm{N}$-telopeptides. ${ }^{28}$ Further, the RCTs were comparison studies evaluating the effects of different doses/formulations of OCs, but two did not include a control group, ${ }^{26}{ }^{28}$ and two used self selected

Table 2 Oxford Centre for Evidence-based Medicine Levels of Evidence

\begin{tabular}{|c|c|}
\hline Level & Evidence \\
\hline la & Systematic review (with homogeneity) of RCTs \\
\hline $1 b$ & Individual RCT with narrow confidence interval \\
\hline 1c & All or none \\
\hline $2 a$ & Systematic review (with homogeneity) of cohort studies \\
\hline $2 b$ & $\begin{array}{l}\text { Individual cohort study (including low quality RCT; e.g. }<80 \% \\
\text { follow up) }\end{array}$ \\
\hline $2 c$ & "Outcomes" research; ecological studies \\
\hline $3 a$ & Systematic review (with homogeneity) of case-control studies \\
\hline $3 b$ & Individual case-control study \\
\hline 4 & Case series (and poor quality cohort and case-control studies) \\
\hline 5 & $\begin{array}{l}\text { Expert opinion without explicit critical appraisal, or based on } \\
\text { physiology, bench research, or first principles }\end{array}$ \\
\hline
\end{tabular}

Table 1 Results from the electronic search strategies

\begin{tabular}{lcccc}
\hline MeSH or keyword & Medline & CINAHL & $\begin{array}{l}\text { CDSR, ACP journal } \\
\text { club, DARE, CCTR }\end{array}$ & SPORTDiscus \\
\hline $\begin{array}{l}1 \text { Bone Density (MeSH) or bone } \\
\text { mineral density (keyword) or bone } \\
\text { density (keyword) }\end{array}$ & 22562 & 2288 & 2473 & 1123 \\
2 Contraceptives, Oral (MeSH) or & 34222 & 3649 & 809 & 197 \\
$\begin{array}{l}\text { ral contraceptive (keyword) } \\
1 \text { and 2 }\end{array}$ & 351 & 212 & 30 & 18 \\
$\begin{array}{l}\text { Limit to English } \\
\text { Total }\end{array}$ & 327 & 212 & 30 & 17 \\
\hline
\end{tabular}

$\mathrm{MeSH}$, Medical subject heading; CINAHL, cumulative index of nursing and allied health literature; CDSR, Cochrane database of systematic reviews; DARE, database of abstracts of reviews of effects; CCTR, Cochrane central register of controlled trials. 
Table 3 Summary of articles reviewed

\begin{tabular}{|c|c|c|c|c|}
\hline & $\begin{array}{l}\text { Healthy } \\
\text { premenopausal }\end{array}$ & $\begin{array}{l}\text { Oligo/amenorrhoeic } \\
\text { premenopausal }\end{array}$ & $\begin{array}{l}\text { Anorexic } \\
\text { premenopausal }\end{array}$ & Perimenopausal \\
\hline Positive effect & $\begin{array}{l}- \\
3 \text { Cohort } \\
7 \text { X-sectional } \\
10\end{array}$ & $\begin{array}{l}2 \text { RCTs } \\
5 \text { Cohort } \\
- \\
7\end{array}$ & $\begin{array}{l}- \\
- \\
2 X \text {-sectional } \\
2\end{array}$ & $\begin{array}{l}1 \mathrm{RCT} \\
4 \text { Cohort } \\
3 \mathrm{X} \text {-sectional } \\
8\end{array}$ \\
\hline No effect & $\begin{array}{l}4 \text { RCTs } \\
9 \text { Cohort } \\
15 \text { X-sectional } \\
1 \text { Case series } \\
29\end{array}$ & $\begin{array}{l}1 \text { RCT } \\
1 \text { Cohort } \\
- \\
- \\
2\end{array}$ & $\begin{array}{l}3 \text { RCTs } \\
1 \text { Cohort } \\
- \\
1 \text { Case series } \\
5\end{array}$ & $\begin{array}{l}- \\
- \\
2 X \text {-sectional } \\
1 \text { Case series } \\
3\end{array}$ \\
\hline Negative effect & $\begin{array}{l}- \\
4 \text { Cohort } \\
3 \text { X-sectional } \\
- \\
7\end{array}$ & $\begin{array}{l}- \\
- \\
- \\
1 \text { Case report } \\
1\end{array}$ & $\begin{array}{l}- \\
1 \text { Cohort } \\
- \\
\overline{1}\end{array}$ & $\begin{array}{l}\overline{-} \\
\overline{-} \\
\overline{-} \\
\overline{0}\end{array}$ \\
\hline Total & 46 & 10 & 8 & 11 \\
\hline
\end{tabular}

Table 4 Healthy premenopausal women: positive effect of oral contraceptives on bone mineral density

\begin{tabular}{|c|c|c|c|c|c|}
\hline Study design & Reference & No of patients & OC exposure & $\begin{array}{l}\text { Measurement of BMD/ } \\
\text { bone metabolism }\end{array}$ & Results \\
\hline \multirow[t]{3}{*}{$\begin{array}{l}\text { Cohort (level 2b, }{ }^{1618} \\
\text { level } 4^{17} \text { ) }\end{array}$} & Recker et al ${ }^{16}$ & 156 college age women & $\begin{array}{l}\text { Current OC users }(n=34) \\
v \text { past users }(n=43) \\
v \text { never }\end{array}$ & $\begin{array}{l}\text { Forearm SPA; spine, } \\
\text { total body DPA }\end{array}$ & $\begin{array}{l}\text { Total body (but not } \\
\text { forearm, spine) BMD } \\
\text { positively correlated with } \\
\text { OC use }\end{array}$ \\
\hline & Berenson et $a l^{7}$ & $\begin{array}{l}155 \text { white, black, Asian, } \\
\text { Hispanic women (ages } \\
18-33 \text { ) in the Armed Forces }\end{array}$ & $\begin{array}{l}35 \mu \mathrm{g} E \mathrm{E}+1 \mathrm{mg} \\
\text { norethindrone }(\mathrm{n}=28) \\
v 30 \mu \mathrm{g} E+0.15 \mathrm{mg} \\
\text { desogestrel }(n=35) \mathrm{v} \\
150 \mathrm{mg} \text { DMPA }(n=33) \\
v \text { control }(n=59) \text { for } \\
12 \text { months }\end{array}$ & Lumbar spine DXA & $\begin{array}{l}\text { Increase in BMD in OC } \\
\text { groups (norethindrone } \\
2.33 \% \text { increase in BMD; } \\
\text { desogesterel O.33\% } \\
\text { increase in BMD) }\end{array}$ \\
\hline & Elgán et $a l^{8}$ & 118 women (ages 18-26) & $\begin{array}{l}\text { Non-smoker/non-OC } \\
\text { users }(n=35) \text { v smoker/ } \\
\text { non-OC user }(n=9) v \\
\text { non-smoker/OC user } \\
(n=57) \text { smoker/OC } \\
\text { user }(n=17)\end{array}$ & $\begin{array}{l}\text { Calcaneus DXA; urinary } \\
\text { D-PYR }\end{array}$ & $\begin{array}{l}\text { OC users had higher } \\
\text { baseline and final BMDs; } \\
\text { smoking was associated } \\
\text { with a larger negative } \\
\text { change in BMD than in } \\
\text { non-smokers; overall, OC } \\
\text { use moderated negative } \\
\text { impact of smoking }\end{array}$ \\
\hline \multirow[t]{7}{*}{ Cross sectional } & $\begin{array}{l}\text { Goldsmith \& } \\
\text { Johnston }\end{array}$ & $\begin{array}{l}2199 \text { pre- and } \\
\text { post-menopausal } \\
\text { women (ages 15-79) }\end{array}$ & $\begin{array}{l}\text { OC users ( } \geqslant 100 \mu \mathrm{g} \\
\text { mestranol, } n=332 ; \\
<100 \mu \mathrm{g} \text { mestranol, } \\
\mathrm{n}=136 ; 50-100 \mu \mathrm{g} \text { EE, } \\
n=83 \text { ) v non-users } \\
(\mathrm{n}=1118)\end{array}$ & $\begin{array}{l}\text { Distal radius }{ }^{125} \text { I photon } \\
\text { absorptiometry }\end{array}$ & $\begin{array}{l}\text { OCs containing } \geqslant 100 \mu \mathrm{g} \\
\text { mestranol increase bone } \\
\text { mineralisation (but OCs } \\
\text { containing } 50-80 \mu \mathrm{g} \\
\text { mestranol or } 50-100 \mu \mathrm{g} \\
\text { EE did not) }\end{array}$ \\
\hline & Lindsay et $a^{p o}$ & 57 women (ages 25-35) & $\begin{array}{l}\text { Ever OC users ( } 30 \text { or } \\
50 \mu \mathrm{g} \mathrm{EE}+\text { norgestrel, } \\
\mathrm{n}=24 \text { ) } \vee \text { never users }\end{array}$ & Lumbar spine DPA & $\begin{array}{l}12 \% \text { higher BMD in ever } \\
\text { OC users than in never } \\
\text { users }\end{array}$ \\
\hline & $\begin{array}{l}\text { Kleerekoper et } \\
a^{P^{1}}\end{array}$ & $\begin{array}{l}2297 \text { women ( } 24 \% \text { pre-, } \\
76 \% \text { post-menopausal) }\end{array}$ & $\begin{array}{l}29.7 \% \text { ever OC users } \\
\text { v } 68.5 \% \text { never OC users } \\
\text { (1.8\% missing) }\end{array}$ & $\begin{array}{l}\text { Forearm SPA, lumbar } \\
\text { spine DPA }\end{array}$ & $\begin{array}{l}\text { Significant association } \\
\text { between duration of } O C \\
\text { use and } B M D \text { (greatest in } \\
\text { those with } \geqslant 10 \text { years } O C \\
\text { use) }\end{array}$ \\
\hline & Laitinen et $a P^{2}$ & $\begin{array}{l}293 \text { Finnish women ( } 186 \\
\text { pre-, } 95 \text { post- menopausal, } \\
12 \text { unknown; ages } 20-76 \text { ) }\end{array}$ & $\begin{array}{l}\text { Premenopausal women: } \\
\text { ever OC users }(n=65) \\
\text { v never users }(n=121)\end{array}$ & $\begin{array}{l}\text { Lumbar spine, proximal } \\
\text { right femur DXA }\end{array}$ & $\begin{array}{l}\text { Significant correlation } \\
\text { between OC use and BMD } \\
\text { in premenopausal women }\end{array}$ \\
\hline & Pasco et a ${ }^{3}$ & $\begin{array}{l}710 \text { Australian women (511 } \\
\text { pre-, } 172 \text { post- menopausal, } \\
27 \text { unknown; ages } 20-69)\end{array}$ & $\begin{array}{l}\text { Ever OC users }(n=579) \\
v \text { never users }(n=131)\end{array}$ & $\begin{array}{l}\text { Lumbar spine, proximal } \\
\text { femur, whole body, distal } \\
\text { forearm DXA }\end{array}$ & $\begin{array}{l}3.3 \% \text { greater mean lumbar } \\
\text { spine BMD in } \\
\text { premenopausal ever OC } \\
\text { users than in never users }\end{array}$ \\
\hline & Cobb et $a^{24}$ & $\begin{array}{l}476 \text { black \& white women } \\
\text { (ages 18-30) }\end{array}$ & $\begin{array}{l}\text { Lifetime month by month } \\
\text { OC history by questionnaire } \\
\text { (quantitative measure) }\end{array}$ & $\begin{array}{l}\text { Spine, whole body, hip } \\
\text { DXA }\end{array}$ & $\begin{array}{l}\text { Significant correlation } \\
\text { between spinal BMD and } \\
\text { cumulative } O C \text { exposure in } \\
\text { white but not black women }\end{array}$ \\
\hline & $\begin{array}{l}\text { Wallace \& } \\
\text { Ballard }^{25}\end{array}$ & $\begin{array}{l}42 \text { white women (ages } \\
19-25 \text { ) }\end{array}$ & $\begin{array}{l}\text { Current OC users }(n=20) \\
v \text { non-users }(n=22)\end{array}$ & $\begin{array}{l}\text { Lumbar spine, total hip } \\
\text { femoral neck, trochanter } \\
\text { total body DXA }\end{array}$ & $\begin{array}{l}\text { Significant correlation } \\
\text { between trochanteric, total } \\
\text { hip BMD and } O C \text { use }\end{array}$ \\
\hline
\end{tabular}

OC, Oral contraceptive; BMD, bone mineral density; SPA, single photon absorptiometry; DPA, dual photon absorptiometry; EE, ethinyl oestradiol; DMPA, deoxymedroxyprogesterone acetate; DXA, dual energy $x$ ray absorptiometry; D-PYR, deoxypyridinoline. 
Table 5 Healthy premenopausal women: no effect of oral contraceptives on bone mineral density

\begin{tabular}{|c|c|c|c|c|c|}
\hline Study design & Reference & No of patients & OC exposure & $\begin{array}{l}\text { Measurement of BMD/ } \\
\text { bone metabolism }\end{array}$ & Results \\
\hline \multirow[t]{4}{*}{$\begin{array}{l}\text { RCT (level } \\
1 b_{1}^{262729} \\
\text { level } 2 b^{28} \text { ) }\end{array}$} & $\begin{array}{l}\text { Castelo-Branco } \\
\text { et } a^{26}\end{array}$ & 67 women (ages 19-29) & $\begin{array}{l}35 \mu \mathrm{gEE}+2 \mu \mathrm{g} \text { CA }(\mathrm{n}=35) \\
v 30 \mu \mathrm{gEE}+150 \mu \mathrm{g} \\
\text { desogestrel }(\mathrm{n}=32) \text { for } \\
24 \text { months }\end{array}$ & DXA & $\begin{array}{l}\text { No changes in BMD from } \\
\text { baseline in either group }\end{array}$ \\
\hline & Nappi et $a P^{7}$ & 60 women (ages 22-34) & $\begin{array}{l}20 \mu \mathrm{g} \mathrm{EE}+75 \mu \mathrm{g} \text { gestodene } \\
(\mathrm{n}=20) \vee 15 \mu \mathrm{gEE}+60 \mu \mathrm{g} \\
\text { gestodene }(\mathrm{n}=20) v \text { control } \\
(\mathrm{n}=20) \text { for } 12 \text { months }\end{array}$ & $\begin{array}{l}\text { Lumbar spine DXA; } \\
\text { urinary PYR, D-PYR, } \\
\text { serum osteocalcin }\end{array}$ & $\begin{array}{l}\text { No changes in BMD from } \\
\text { baseline in any group; } \\
\text { decrease in PYR, D-PYR in OC } \\
\text { treated groups suggesting } \\
\text { decreased resorption }\end{array}$ \\
\hline & Endrikat et $a{ }^{28}$ & 48 women (ages 20-38) & $\begin{array}{l}30 \mu \mathrm{g} \mathrm{EE}+150 \mu \mathrm{g} \\
\text { levonorgestrel }(n=25) \\
v 20 \mu \mathrm{g} \mathrm{EE}+100 \mu \mathrm{g} \\
\text { levonorgestrel }(n=23) \\
\text { for } 36 \text { months }\end{array}$ & $\begin{array}{l}\text { Lumbar spine qCT; } \\
\text { serum BSAP, } \\
\text { urinary NTx }\end{array}$ & $\begin{array}{l}\text { No changes in BMD from } \\
\text { baseline in either group; } \\
\text { decrease in NTx in both } \\
\text { groups (suggesting decreased } \\
\text { resorption) }\end{array}$ \\
\hline & Nappi et $a f^{p}$ & 71 women (ages 22-34) & $\begin{array}{l}30 \mu \mathrm{g} \mathrm{EE}+3 \mathrm{mg} \text { drospirenone } \\
(n=24) \vee 30 \mu \mathrm{gE}+75 \mu \mathrm{g} \\
\text { gestodene }(n=24) \vee \text { control } \\
(n=23) \text { for } 12 \text { months }\end{array}$ & $\begin{array}{l}\text { Lumbar spine DXA; } \\
\text { serum \& urinary } \mathrm{Ca}^{2+} \\
\text { serum osteocalcin, } \\
\text { urinary PYR, D-PYR }\end{array}$ & $\begin{array}{l}\text { Decrease in PYR, D-PYR in } \\
\text { both OC treated groups from } \\
\text { baseline (suggesting } \\
\text { decreased resorption); trend } \\
\text { to increased BMD in } \\
\mathrm{EE}+\text { drospirenone group }\end{array}$ \\
\hline \multirow[t]{3}{*}{$\begin{array}{l}\text { Cohort } \\
\text { (level 2b) }\end{array}$} & Mazess \& Barden ${ }^{30}$ & 300 women (ages 20-39) & $\begin{array}{l}50 \% \text { past/current } O C \text { users, } \\
50 \% \text { never users }\end{array}$ & $\begin{array}{l}\text { Lumbar spine DPA, } \\
\text { radius SPA }\end{array}$ & $\begin{array}{l}\text { No association between } O C \\
\text { use and } B M D\end{array}$ \\
\hline & Cromer ef $a f^{\beta 1}$ & 48 women (ages 12-21) & $\begin{array}{l}30 \mu \mathrm{g} \mathrm{EE}+150 \mu \mathrm{g} \text { desogestrel } \\
(n=9) v \text { Norplant }(n=7) v \\
\text { Depo-Provera }(n=15) v \text { control } \\
(n=17) \text { for } 12 \text { months }\end{array}$ & Lumbar spine DXA & $\begin{array}{l}\text { No significant difference } \\
\text { between change in BMD in } \\
O C \text { treated group ( } 1.5 \% \\
\text { increase in BMD) } v \text { control } \\
(2.9 \% \text { increase in BMD) }\end{array}$ \\
\hline & Lloyd et $a^{\beta 2}$ & $\begin{array}{l}62 \text { white women (followed } \\
\text { from age } 12-20 \text { years) }\end{array}$ & $\begin{array}{l}\text { OC users ("low dose } \\
\text { monophasic") }(n=28) \\
v \text { non-users }(n=34)\end{array}$ & Proximal femur DXA & $\begin{array}{l}\text { No effect of } O C \text { treatment on } \\
\text { peak bone mass or rate of } \\
\text { acquisition }\end{array}$ \\
\hline \multirow[t]{6}{*}{$\begin{array}{l}\text { Cohort } \\
\text { (level } 4,^{3334} \\
\text { level } 2 b^{35-38} \text { ) }\end{array}$} & Reed et $a \beta^{33}$ & 245 women (ages 18-39) & $\begin{array}{l}\text { Current OC users ( } 80 \% \text { on } \\
30-35 \mu \mathrm{gEE})(n=89) v \\
\text { control }(n=156)\end{array}$ & $\begin{array}{l}\text { Lumbar spine, } \\
\text { proximal femur, total } \\
\text { body DXA }\end{array}$ & $\begin{array}{l}\text { No change in BMD from } \\
\text { baseline in either group }\end{array}$ \\
\hline & ${\text { Lara-Torre ef } a^{\beta 4}}^{\beta 4}$ & 148 women (ages 11-21) & $\begin{array}{l}\text { New OC users }(n=71) v \\
\text { new DMPA users }(n=58) v \\
\text { control }(n=19) \text { over } 24 \\
\text { months }\end{array}$ & Lumbar spine DXA & $\begin{array}{l}\text { No change in BMD from } \\
\text { baseline in } O C \text { users }\end{array}$ \\
\hline & Lloyd et al ${ }^{\beta 5}$ & 80 women (ages 12-22) & $\begin{array}{l}\text { OC users (for } \geqslant 6 \text { months, } \\
\text { and still using at age } 22) \\
(n=33) \vee \text { non-users }(n=17)\end{array}$ & $\begin{array}{l}\text { Total body, bilateral } \\
\text { proximal femur DXA }\end{array}$ & $\begin{array}{l}\text { No difference in BMD } \\
\text { between } O C \text { users and non- } \\
\text { users }\end{array}$ \\
\hline & Berenson et $a^{\beta 6}$ & 191 women (ages 18-33) & $\begin{array}{l}\mathrm{OC}(35 \mu \mathrm{g} \mathrm{EE}+1 \mathrm{mg} \\
\text { norethindrone or } 30 \mu \mathrm{g} \\
\mathrm{EE}+0.15 \mathrm{mg} \text { desogestrel) } \\
(\mathrm{n}=86) \mathrm{v} \text { DMPA ( } \mathrm{n}=47) \\
v \text { control }(\mathrm{n}=58) \text { for } 24 \text { months }\end{array}$ & Lumbar spine DXA & $\begin{array}{l}\text { No difference in BMD change } \\
\text { from baseline between } O C \\
\text { groups and control (decrease } \\
\text { in BMD from baseline in } \\
\text { DMPA group } v \text { control) }\end{array}$ \\
\hline & Paoletti ef $\left.a\right|^{\beta 7}$ & 54 women (ages 20-30) & $\begin{array}{l}30 \mu \mathrm{g} \mathrm{EE}+3 \mathrm{mg} \text { drospirenone } \\
(\mathrm{n}=28) \vee \text { control }(n=26) \text { for } \\
6 \text { months }\end{array}$ & $\begin{array}{l}\text { Heel DXA+laser; } \\
\text { serum osteocalcin, } \\
\text { BSAP, urinary PYR, } \\
\text { D-PYR }\end{array}$ & $\begin{array}{l}\text { No change in BMD from } \\
\text { baseline in any group; } \\
\text { decrease in osteocalcin, } \\
\text { BSAP, PYR in OC group } \\
\text { (suggesting decreased bone } \\
\text { turnover) }\end{array}$ \\
\hline & Rome ef $a^{\beta 8}$ & 370 women (ages 12-18) & $\begin{array}{l}20 \mu \mathrm{g} \mathrm{EE}+100 \mu \mathrm{g} \\
\text { levonorgestrel }(\mathrm{n}=165) \\
v \text { DMPA }(n=53) v \text { control } \\
(n=152) \text { for } 12 \text { months }\end{array}$ & $\begin{array}{l}\text { Lumbar spine, hip } \\
\text { DXA; serum BSAP, } \\
\text { urinary D-PYR }\end{array}$ & $\begin{array}{l}\text { Increase in BSAP in control } v \\
O C \text {, but no difference in BMD } \\
\text { between groups }\end{array}$ \\
\hline \multirow[t]{6}{*}{$\begin{array}{l}\text { Cross } \\
\text { sectional }\end{array}$} & Sowers et $a{ }^{\beta 9}$ & 86 women (ages 20-35) & $\begin{array}{l}\text { OC users (for }>2 \text { months) } \\
(n=78) \text { v non-users }(n=8)\end{array}$ & $\begin{array}{l}\text { Bone mass by }{ }^{125} \\
\text { photon absorptiometry }\end{array}$ & $\begin{array}{l}\text { No difference in bone mass } \\
\text { between ever } v \text { never users or } \\
\text { between current } v \text { past users }\end{array}$ \\
\hline & Hreschyshyn et a ${ }^{40}$ & $\begin{array}{l}352 \text { women (pre- and post- } \\
\text { menopausal; ages } 24-79 \text { ) }\end{array}$ & $\begin{array}{l}\text { Ever OC users }(n=116) \\
v \text { never users }(n=236)\end{array}$ & $\begin{array}{l}\text { Lumbar spine, femoral } \\
\text { neck DPA }\end{array}$ & $\begin{array}{l}\text { No difference in } B M D \\
\text { between ever } O C \text { users and } \\
\text { never users }\end{array}$ \\
\hline & Lloyd et a ${ }^{41}$ & 25 women & $\begin{array}{l}\text { OC users (minimum } 50 \mu \mathrm{g} \\
\text { mestranol/day) }(n=14) \\
v \text { non-users }(n=11)\end{array}$ & Lumbar spine qCT & $\begin{array}{l}\text { No difference in BMD } \\
\text { between } O C \text { users and non- } \\
\text { users }\end{array}$ \\
\hline & Stevenson et $\left.a\right|^{42}$ & $\begin{array}{l}284 \text { white women ( } 112 \text { pre-, } \\
172 \text { post- menopausal) }\end{array}$ & OC users $v$ non-users & $\begin{array}{l}\text { Lumbar spine, } \\
\text { proximal femur DPA }\end{array}$ & $\begin{array}{l}\text { No association between } O C \\
\text { use and } B M D \text { in } \\
\text { premenopausal women }\end{array}$ \\
\hline & Hall et $\left.a\right|^{43}$ & $\begin{array}{l}165 \text { women (pre- and post- } \\
\text { menopausal; ages } 4-80 \text { ) }\end{array}$ & $\begin{array}{l}\text { Ever OC users }(n=69) \\
v \text { never users }(n=96)\end{array}$ & Lumbar spine DXA & $\begin{array}{l}\text { No difference in BMD } \\
\text { between ever OC users and } \\
\text { non-users in any age group }\end{array}$ \\
\hline & Murphy et $a^{\mu 4}$ & $\begin{array}{l}841 \text { women ( } 229 \text { pre--, } \\
\text { perimenopausal, } 583 \\
\text { postmenopausal, } 29 \\
\text { unknown) }\end{array}$ & $\begin{array}{l}\text { Ever OC users ( } n=159 \\
\text { pre-, perimenopausal; } \\
n=182 \text { postmenopausal; } \\
n=11 \text { unknown) } v \text { never } \\
\text { users ( } n=70 \text { pre-, } \\
\text { peri-menopausal; } n=401 \\
\text { postmenopausal; } n=18 \\
\text { unknown) }\end{array}$ & $\begin{array}{l}\text { Lumbar spine, } \\
\text { hip DXA }\end{array}$ & $\begin{array}{l}\text { No difference in BMD } \\
\text { between ever OC users and } \\
\text { non-users }\end{array}$ \\
\hline
\end{tabular}




\begin{tabular}{|c|c|c|c|c|c|}
\hline Study design & Reference & No of patients & OC exposure & $\begin{array}{l}\text { Measurement of BMD/ } \\
\text { bone metabolism }\end{array}$ & Results \\
\hline & Garnero et $a l^{\mu 5}$ & 208 women (ages 35-49) & $\begin{array}{l}\text { OC users (combined pills } \\
\text { with } 30 \mu \mathrm{gE}, n=41 ; \\
\text { combined pills with } 50 \mu \mathrm{g} \\
\mathrm{EE}, \mathrm{n}=3 \text {; sequential combined } \\
\text { pills, } n=5 \text {; progestative } \\
\text { contraceptives, } \mathrm{n}=3 \text { ) } \\
\text { (total } \mathrm{n}=52 \text { ) } v \text { non-users } \\
\text { ( } \mathrm{n}=156 \text { ) }\end{array}$ & $\begin{array}{l}\text { Lumbar spine, total } \\
\text { body, hip, distal } \\
\text { radius DXA; serum } \\
\text { osetocalcin, BSAP, C } \\
\text { terminal propeptide } \\
\text { of type I collagen, } \\
\text { urinary NTx and PYR }\end{array}$ & $\begin{array}{l}\text { No difference in } B M D \\
\text { between } O C \text { users and non- } \\
\text { users; decrease in markers of } \\
\text { both formation and resorption } \\
\text { in } O C \text { users v non-users } \\
\text { (suggesting decreased bone } \\
\text { turnover) }\end{array}$ \\
\hline & Ulrich et $a l^{\mu 6}$ & 25 women (mean age 41 ) & Ever $O C$ users $v$ never users & $\begin{array}{l}\text { Axial, peripheral } \\
\text { BMD by DXA }\end{array}$ & $\begin{array}{l}\text { No difference in BMD } \\
\text { between ever OC users and } \\
\text { never users }\end{array}$ \\
\hline & Petitti et $a^{\mu 7}$ & 2474 women (ages $30-34$ ) & $\begin{array}{l}\text { Ever OC users }(82 \%>30 \\
\text { but }<50 \mu \mathrm{g} \text { oestrogen, } 15 \% \\
\geqslant 50 \mu \mathrm{g} \text { oestrogen, }<1 \% \\
<30 \mu \mathrm{g} \text { oestrogen, } 2 \% \\
\text { unknown dose) }(\mathrm{n}=819) \\
v \text { ever DMPA users }(\mathrm{n}=350) \\
v \text { ever levonorgestrel users } \\
(\mathrm{n}=610) \text { v control }(\mathrm{n}=695)\end{array}$ & $\begin{array}{l}\text { Distal radius, } \\
\text { midshaft ulna SXA }\end{array}$ & $\begin{array}{l}\text { No difference in BMD } \\
\text { between ever users of } \\
\text { hormonal contraception } v \\
\text { never users }\end{array}$ \\
\hline & Ott et $a l^{48}$ & 227 women (ages 18-39) & $\begin{array}{l}\text { OC users }(53.6 \% 35 \mu \mathrm{g} \\
\mathrm{EE}+0.5-1 \mathrm{mg} \text { norethindrone, } \\
18 \% 35 \mu \mathrm{gEE}+1 \mathrm{mg} \\
\text { levonorgestrel or } 1 \mathrm{mg} \\
\text { ethynodiol diacetate, } 13.7 \% \\
30 \mu \mathrm{g} \mathrm{EE}+1.5 \mathrm{mg} \\
\text { norethindrone, } 9.7 \% 20 \mu \mathrm{g} \\
\mathrm{EE}+\text { levonorgestrel or } \\
\text { norethindrone) }(\mathrm{n}=39) \\
v \mathrm{DMPA}(\mathrm{n}=116) \\
v \text { control }(\mathrm{n}=72)\end{array}$ & $\begin{array}{l}\text { Lumbar spine, } \\
\text { total body, total hip } \\
\text { DXA; serum } \mathrm{Ca}^{2+} \text {, } \\
\text { PTH, osteocalcin, } \\
\text { urinary NTx }\end{array}$ & $\begin{array}{l}\text { No difference in BMD } \\
\text { between any of the groups; } \\
\text { decrease in osteocalcin and } \\
\text { NTx in OC users than in non- } \\
\text { users (suggesting decreased } \\
\text { bone turnover) }\end{array}$ \\
\hline & Perotti et a ${ }^{\mu 9}$ & 189 women (ages 30-34) & $\begin{array}{l}\text { OC users (for } \geqslant 2 \text { years) } \\
(n=63) v \text { DMPA users } \\
\text { (for } \geqslant 2 \text { years) }(n=63) \\
v \text { control (no hormonal } \\
\text { contraception) }(n=63)\end{array}$ & $\begin{array}{l}\text { Non-dominant } \\
\text { radius SXA }\end{array}$ & $\begin{array}{l}\text { No difference in BMD } \\
\text { between any of the groups }\end{array}$ \\
\hline & Hawker et $a P^{50}$ & 830 women (ages 19-35) & $\begin{array}{l}\text { Current OC users }(n=223) \\
v \text { past OC users }(n=512) \\
v \text { never users }(n=95)\end{array}$ & $\begin{array}{l}\text { Non-dominant } \\
\text { radius SXA }\end{array}$ & $\begin{array}{l}\text { No association between } O C \\
\text { use and BMD }\end{array}$ \\
\hline & $\begin{array}{l}\text { Wanichsetakul } \\
\text { ef } a^{p^{51}}\end{array}$ & 155 women (ages 30-34) & $\begin{array}{l}\text { OC users }(n=59) \vee \text { DMPA } \\
(n=34) \vee \text { control }(n=62)\end{array}$ & $\begin{array}{l}\text { Lumbar spine, femoral } \\
\text { neck, Ward's triangle, } \\
\text { greater trochanter, } \\
\text { radius, ulna DPA }\end{array}$ & $\begin{array}{l}\text { No difference in BMD } \\
\text { between } O C \text { users and } \\
\text { control }\end{array}$ \\
\hline & Afghani et $a^{\beta^{2}}$ & $\begin{array}{l}39 \text { Hispanic pre-/ } \\
\text { peri-menopausal women } \\
\text { (ages 22-51) }\end{array}$ & Current $\mathrm{OC}$ user $v$ non-user & Whole body DXA & $\begin{array}{l}\text { No relation between current } \\
\text { OC use and BMD (but no info } \\
\text { re duration of use, past use, } \\
\text { dose, etc) }\end{array}$ \\
\hline & Meyer et $a^{33}$ & $\begin{array}{l}61 \text { women ( } 40 \text { athletes (19 } \\
\text { eumenorrhoeic, } 21 \\
\text { oligoamenorrhoeic) } 21 \\
\text { eumenorrhoeic non-athletes; } \\
\text { mean age } 26 \text { years) }\end{array}$ & Current $\mathrm{OC}$ user $v$ non-user & $\begin{array}{l}\text { Areal BMD of whole } \\
\text { body, lumbar spine, } \\
\text { proximal femur, } \\
\text { femoral neck, greater } \\
\text { trochanter }\end{array}$ & $\begin{array}{l}\text { No association between } O C \\
\text { use and areal BMD in athlete } \\
\text { group }\end{array}$ \\
\hline $\begin{array}{l}\text { Case series } \\
\text { (level 4) }\end{array}$ & Mais et $a^{54}$ & 19 women (ages 20-30) & $\begin{array}{l}20 \mu \mathrm{g} \mathrm{EE}+0.15 \mathrm{mg} \\
\text { desogestrel for } 12 \mathrm{months}\end{array}$ & $\begin{array}{l}\text { Distal radius DPA; } \\
\text { serum BSAP, urinary } \\
\text { hydroxyproline: } \mathrm{Cr}\end{array}$ & $\begin{array}{l}\text { NS increase in BMD; } \\
\text { decrease in BSAP, } \\
\text { hydroxyproline (suggesting } \\
\text { decreased bone turnover) }\end{array}$ \\
\hline \multicolumn{6}{|c|}{$\begin{array}{l}\text { OC, Oral contraceptive; BMD, bone mineral density; RCT, randomised controlled trial; EE, ethinyl oestradiol; CA, cyproterone acetate; DXA, dual energy } x \text { ray } \\
\text { absorptiometry; PYR, pyridinoline; D-PYR, deoxypyridinoline; qCT, quantitative computed tomography; BSAP, bone specific alkaline phosphatase; NTx, N- } \\
\text { telopeptides; DPA, dual photon absorptiometry; SPA, single photon absorptiometry; DMPA, deoxymedroxyprogesterone acetate; SXA, single energy } x \text { ray } \\
\text { absorptiometry. PTH parathyroid hormone: } \mathrm{Cr} \text { creatinine: NS, non-sianificant. }\end{array}$} \\
\hline
\end{tabular}

control groups choosing not to receive contraception, ${ }^{27}{ }^{29}$ which may have affected the validity of the results. No RCT showed a negative effect. But notably, two cohort studies ${ }^{56} 57$ and one cross sectional study ${ }^{59}$ examined the combination of exercise and OCs on BMD. As previously discussed, exercise is believed to have a positive effect on $\mathrm{BMD}$, according to Frost's mechanostat theory. ${ }^{14}$ However, Burr et al ${ }^{56}$ showed that either exercise or OCs alone was associated with a suppression of the normal increase in femoral neck BMD in women 18-31 years old, but the combination of exercise and OCs together had a less suppressive effect than either alone. Similarly, Weaver et al ${ }^{57}$ suggested that exercise in combination with OCs compromised attainment of peak spinal BMD. Hartard et al $^{59}$ reported that women with long term exercise and short term OC use had the highest lumbar spine and femoral neck BMD, whereas women with long term exercise and long term OC use had comparable BMD values to women with short term exercise and either long or short term OC use, suggesting that OCs offset the beneficial effects of exercise on BMD.

\section{Oligo/amenorrhoeic premenopausal women}

Ten studies on oligo/amenorrhoeic premenopausal women were reviewed. Menstrual irregularities were classified as "hypothalamic" oligo/amenorrhoea-that is, functional menstrual irregularity-or that occurring in the absence of an organic cause (except for two cohort studies which included subjects with primary ovarian failure ${ }^{66}$ and from a variety of unspecified causes $\left.{ }^{65}\right)$. Although these conditions often occur in athletic females, as previously discussed, it is the energy 
Table 6 Healthy premenopausal women: negative effect of oral contraceptives on bone mineral density

\begin{tabular}{|c|c|c|c|c|c|}
\hline Study design & Reference & No of patients & OC exposure & $\begin{array}{l}\text { Measurement of BMD/ } \\
\text { bone metabolism }\end{array}$ & Results \\
\hline \multirow[t]{4}{*}{$\begin{array}{l}\text { Cohort } \\
\text { (level } 2 b_{1}^{58} \\
\text { level } 4^{55-57} \text { ) }\end{array}$} & Polatti et $a^{55}$ & $\begin{array}{l}200 \text { women } \\
\text { (ages 19-22) }\end{array}$ & $\begin{array}{l}20 \mu \mathrm{g} \mathrm{EE}+0.15 \mathrm{mg} \\
\text { desogestrel }(n=100) \\
v \text { control }(n=100) \text { for } \\
60 \text { months }\end{array}$ & $\begin{array}{l}\text { Lumbar spine DXA; serum } \\
\text { BSAP, urinary } \\
\text { hydroxyproline: } \mathrm{Cr}\end{array}$ & $\begin{array}{l}\text { No change in BMD in treated group } v \\
\text { increase in BMD in control group; no } \\
\text { change in BSAP or hydroxyproline } \\
\text { levels in either group }\end{array}$ \\
\hline & Burr et $a^{56}$ & $\begin{array}{l}46 \text { women } \\
\text { (ages 18-31) }\end{array}$ & $\begin{array}{l}\text { Non-exercisers/non-OC users } \\
(n=10) v \text { non-exercisers }+ \\
\leqslant 50 \mu \mathrm{gEE}(n=13) v \\
\text { exercisers/non-OC users } \\
(n=8) v \text { exercisers }+\leqslant 50 \mu \mathrm{g} \\
\mathrm{EE}(\mathrm{n}=15)\end{array}$ & $\begin{array}{l}\text { Femoral neck DXA; serum } \\
\text { osteocalcin, BSAP, acid } \\
\text { phosphatase, urinary } \\
\text { hydroxyproline:Cr }\end{array}$ & $\begin{array}{l}\text { Either } O C \text { use or exercise alone is } \\
\text { associated with suppression of the } \\
\text { normal increase in femoral neck } \\
\text { bone mass/mechanical strength; } \\
\text { combination of } O C \text { use and exercise } \\
\text { has less suppressive effect than either } \\
\text { alone }\end{array}$ \\
\hline & Weaver et $a P^{p 7}$ & $\begin{array}{l}179 \text { women } \\
\text { (ages 18-31) }\end{array}$ & $\begin{array}{l}\text { Non-exercisers/non-OC users } \\
(n=40) v \text { non-exercisers }+ \\
\leqslant 50 \mu \mathrm{gEE}(\mathrm{n}=37) v \\
\text { exercisers } / \text { non-OC users } \\
(\mathrm{n}=37) v \text { exercisers }+\leqslant 50 \mu \mathrm{g} \\
\mathrm{EE}(\mathrm{n}=40)\end{array}$ & $\begin{array}{l}\text { Lumbar spine, total body } \\
\text { total hip DXA; radius SPA; } \\
\text { serum osteocalcin, BSAP } \\
\text { acid phosphatase, urinary } \\
\text { hydroxyproline:Cr }\end{array}$ & $\begin{array}{l}\text { Significant interaction between } O C \\
\text { use and exercise, such that a } \\
\text { combination of } O C \text { use and exercise } \\
\text { compromises attainment of peak } \\
\text { spinal BMD }\end{array}$ \\
\hline & Cromer et $\left.a\right|^{58}$ & $\begin{array}{l}215 \text { women } \\
\text { (ages } 12-18 \text { ) }\end{array}$ & $\begin{array}{l}20 \mu \mathrm{g} \mathrm{EE}+100 \mu \mathrm{g} \\
\text { levonorgestrel }(n=79) \\
v \text { DMPA }(n=29) v \text { control } \\
(n=107) \text { over } 12 \text { months }\end{array}$ & $\begin{array}{l}\text { Lumbar spine, total hip, } \\
\text { femoral neck, Ward's } \\
\text { triangle, trochanter DXA }\end{array}$ & $\begin{array}{l}\text { Increase in spine and hip BMD in } \\
\text { both } O C \text { and control groups, but } \\
\text { increase in } O C \text { group was } \\
\text { significantly less than that in control } \\
\text { group }\end{array}$ \\
\hline \multirow[t]{3}{*}{ Cross sectional } & Hartard et $a f^{59}$ & $\begin{array}{l}128 \text { women } \\
\text { (ages } 20-35 \text { ) }\end{array}$ & $\begin{array}{l}\text { Long term exercise/short term } \\
\text { use }(n=30) v \text { long term } \\
\text { exercise/long term OC use } \\
(n=37) v \text { short term exercise/ } \\
\text { long term OC use }(n=31) \\
v \text { short term exercise/ } \\
\text { short term OC use }(n=30)\end{array}$ & $\begin{array}{l}\text { Lumbar spine, femoral } \\
\text { neck DXA }\end{array}$ & $\begin{array}{l}\text { Highest BMD in long term exercise/ } \\
\text { short term OC use group; no } \\
\text { differences in mean BMD between } \\
\text { short term exercise/long term OC use } \\
\text { and short term exercise/short term } \\
O C \text { use; overall, OC use counteracts } \\
\text { beneficial effect of exercise on BMD? }\end{array}$ \\
\hline & Prior et $a^{k 0}$ & $\begin{array}{l}524 \text { women } \\
\text { (ages } 25-45 \text { ) }\end{array}$ & $\begin{array}{l}\text { Ever } O C \text { users (for } \geqslant 3 \text { months) } \\
\text { ( } n=454) \text { v never users ( } 0 \text { to } \\
<3 \text { months) }(n=70)\end{array}$ & $\begin{array}{l}\text { Lumbar spine, proximal } \\
\text { femur DXA }\end{array}$ & $\begin{array}{l}\text { Decrease in lumbar spine, trochanter } \\
B M D \text { in ever } O C \text { users } v \text { never users }\end{array}$ \\
\hline & Hartard et $a^{\beta 1}$ & $\begin{array}{l}69 \text { female } \\
\text { endurance } \\
\text { athletes } \\
\text { (ages 18-35) }\end{array}$ & $\begin{array}{l}\text { OC group (use for }>3 \text { years in } \\
\text { women }<22 \text { years old or use } \\
\text { for }>50 \% \text { of time after } \\
\text { menarche in women age } \\
22-35)(n=31) v \text { control } \\
(n=38)\end{array}$ & Lumbar spine, hip DXA & $\begin{array}{l}\text { OC users had } 7.9 \% \text { lower lumbar } \\
\text { spine and } 8.8 \% \text { lower proximal } \\
\text { femur BMD than control }\end{array}$ \\
\hline
\end{tabular}

OC, Oral contraceptive; BMD, bone mineral density; EE, ethinyl oestradiol; DXA, dual energy $x$ ray absorptiometry; BSAP, bone specific alkaline phosphatase; Cr, creatinine; SPA, single photon absorptiometry; DMPA, deoxymedroxyprogesterone acetate.

deficit, rather than the activity itself, that leads to the menstrual dysfunction. In the reproductive literature, eumenorrhoea is defined as cycles with intervals of 25-34 days, whereas oligomenorrhoea typically refers to menstrual cycles longer than 35 days. The term amenorrhoea (secondary) connotes a persistent absence of menstrual cycles, commonly for three or more months after the establishment of regular menses. However, confusion often arises when comparing studies, because of the inconsistency of definitions, particularly in earlier research.

Of the 10 studies of OC and other hormone replacement in this population, seven (two RCTs, ${ }^{62}{ }^{63}$ five cohort ${ }^{64-68}$ ) showed a positive effect, two (one RCT, ${ }^{69}$ one cohort $^{70}$ ) showed no effect, and one case report ${ }^{71}$ showed a negative effect on BMD. In all studies that compared baseline BMDs with that of healthy controls or age matched reference values, baseline BMDs were significantly lower in the oligo/amenorrhoeic subjects. ${ }^{65-71}$ Hergenroeder $e t ~ a l^{62}$ showed an increase in total body and lumbar spine BMD with OCs, compared with medroxyprogesterone or placebo. Although well designed, this was a small study with only five subjects per treatment group, followed over a 12 month time span. In a somewhat larger study (18-24 subjects per group), Castelo-Branco et a $l^{63}$ examined the effects of two doses $(20$ or $30 \mu \mathrm{g})$ of ethinyl oestradiol-containing OCs on lumbar spine BMD. Both doses increased $\mathrm{BMD}$, whereas the $\mathrm{BMD}$ of the control group decreased. ${ }^{63}$ Conversely, Gibson ${ }^{69}$ showed that lumbar spine and hip BMD did not significantly change with OCs, calcium carbonate, or control. This trial was conducted over 18 months; however, data from only nine months were reported because of a high dropout rate ${ }^{69}$ Further, the OC treated group in this study did show a non-significant increase in BMD after nine months. ${ }^{69}$ No RCT showed a negative effect of OC treatment on BMD.

\section{Anorexic premenopausal women}

Eight studies on premenopausal women with anorexia nervosa were reviewed. Subjects were defined as having anorexia nervosa by either the Diagnostic and statistical manual of mental disorders (DSM)-IIIR or DSM-IV criteria (except for two studies, ${ }^{73}{ }^{79}$ in which the criteria used were not explicitly stated). Two cross sectional studies ${ }^{72}{ }^{73}$ showed a positive effect, five studies (three RCTs, ${ }^{74-76}$ one cohort, ${ }^{77}$ one case series $^{78}$ ) showed no effect, and one cohort study ${ }^{79}$ showed a negative effect. Klibanski et $a l^{74}$ found no overall change in lumbar spine BMD from baseline in either the oestrogen treated or control group. However, the effect of oestrogen on BMD was greatest in patients with the lowest initial body weight, and diminished with increasing patient weight. ${ }^{74}$ Control patients with a baseline body weight $<70 \%$ of ideal experienced a significant decrease in BMD, whereas oestrogen treated patients with baseline body weight $<70 \%$ of ideal did not experience any significant change in BMD, suggesting that, in anorexic women, oestrogen may have a body weight dependent effect on BMD. ${ }^{74}$ Gordon et al ${ }^{75}$ showed no effect of either dehydroepiandrosterone or OCs on total hip BMD in anorexic women. In both groups, non-significant increases in lumbar BMD and significantly decreased $\mathrm{N}$ telopeptide concentrations were reported. ${ }^{75}$ Grinspoon et al ${ }^{76}$ examined the effect of OCs, recombinant human insulin-like 
Table 7 Oligo/amenorrhoeic premenopausal women: positive effect of oral contraceptives on bone mineral density

\begin{tabular}{|c|c|c|c|c|c|}
\hline Study design & Reference & No of patients & $\mathrm{OC}$ exposure & $\begin{array}{l}\text { Measurement of BMD/ } \\
\text { bone metabolism }\end{array}$ & Results \\
\hline \multirow[t]{2}{*}{ RCT (level 1b) } & $\begin{array}{l}\text { Hergenroeder } \\
\text { et } a^{k^{2}}\end{array}$ & $\begin{array}{l}24 \text { women with } \\
\text { hypothalamic } \\
\text { amenorrhoea } \\
\text { (ages 14-28) }\end{array}$ & $\begin{array}{l}35 \mu \mathrm{gEE}+0.5-1 \mathrm{mg} \\
\text { norethindrone }(\mathrm{n}=5) \text { v } 10 \mathrm{mg} \\
\text { medroxyprogesterone }(\mathrm{n}=5) \mathrm{v} \\
\text { placebo }(\mathrm{n}=5) \text { for } 12 \text { months }\end{array}$ & $\begin{array}{l}\text { Lumbar spine, } \\
\text { total body, } \\
\text { femoral neck DXA }\end{array}$ & $\begin{array}{l}\text { Increase in lumbar spine \& total body } \\
B M D \text { in } O C \text { treated group } v \text { placebo; } \\
\text { no change in } B M D \text { at any site in } \\
\text { medroxyprogesterone treated group }\end{array}$ \\
\hline & $\begin{array}{l}\text { Castelo-Branco } \\
\text { et } a^{k^{3}}\end{array}$ & $\begin{array}{l}64 \text { women with } \\
\text { hypothalamic } \\
\text { oligomenorrhoea } \\
\text { (ages 19-35) }\end{array}$ & $\begin{array}{l}30 \mu \mathrm{g} \mathrm{EE}+0.15 \mathrm{mg} \text { desogestrel } \\
(\mathrm{n}=24) \vee 20 \mu \mathrm{gE}+0.15 \mathrm{mg} \\
\text { desogestrel }(\mathrm{n}=22) \vee \text { control } \\
(\mathrm{n}=18) \text { for } 12 \text { months }\end{array}$ & Lumbar spine DXA & $\begin{array}{l}\text { Increase in lumbar spine BMD in both } \\
\text { OC treated groups; decrease in BMD } \\
\text { in control group }\end{array}$ \\
\hline \multirow[t]{5}{*}{$\begin{array}{l}\text { Cohort (level } \\
2 b^{646768} \\
\text { level } 4^{65} 66 \text { ) }\end{array}$} & De Creé et $\left.a\right|^{\beta 4}$ & $\begin{array}{l}11 \text { sportswomen } \\
\text { with athletic } \\
\text { menstrual } \\
\text { irregularity } \\
\text { (ages 18-29) }\end{array}$ & $\begin{array}{l}50 \mu \mathrm{g} \mathrm{EE}+2 \mathrm{mg} \text { cyproterone } \\
\text { acetate }(n=7) \vee \text { control } \\
(n=4) \text { for } 8 \text { months }\end{array}$ & $\begin{array}{l}\text { Lumbar spine DPA, } \\
\text { radius SPA }\end{array}$ & $\begin{array}{l}9.5 \% \text { increase in lumbar spine BMD } \\
\text { in OC treated group }\end{array}$ \\
\hline & Gulekli et a ${ }^{k 5}$ & $\begin{array}{l}85 \text { women with } \\
\text { past }(n=33) \text { or } \\
\text { current }(n=52) \\
\text { history of } \\
\text { amenorrhoea } \\
\text { (ages 17-40) }\end{array}$ & $\begin{array}{l}\text { Synthetic oestrogens }(10-50 \mu \mathrm{g} \\
\text { EE) }(\mathrm{n}=40) \vee \text { natural oestrogens } \\
\text { (Premarin or oestradiol valerate) } \\
(\mathrm{n}=10) \vee 50 \mathrm{mg} \text { transdermal } \\
\text { estradiol }(n=8) \vee \text { bromocriptine } \\
(\mathrm{n}=9) \vee \text { weight gain }(n=6) v \\
\text { control (untreated) }(n=12) \text { for } 3 \\
\text { years }\end{array}$ & Lumbar spine DXA & $\begin{array}{l}\text { Increase in BMD in all treatment } \\
\text { groups, but weight gain was most } \\
\text { effective treatment; NS decrease in } \\
\text { BMD in control group }\end{array}$ \\
\hline & Haenggi et al ${ }^{\beta \sigma}$ & $\begin{array}{l}21 \text { women with } \\
\text { hypothalamic or } \\
\text { ovarian } \\
\text { amenorrhoea, } \\
123 \text { healthy } \\
\text { controls } \\
\text { (ages 18-45) }\end{array}$ & $\begin{array}{l}30 \mu \mathrm{g} E E+0.15 \mathrm{mg} \text { desogestrel } \\
(n=15) v \text { control }(n=123) \text { for } \\
24 \text { months }\end{array}$ & $\begin{array}{l}\text { Lumbar spine, } \\
\text { proximal femur DXA }\end{array}$ & $\begin{array}{l}\text { Initial BMD was lower in } \\
\text { amenorrhoeic women than in healthy } \\
\text { women; increase in lumbar spine, } \\
\text { Ward's triangle BMD in OC treated } \\
\text { group }\end{array}$ \\
\hline & Cumming $^{67}$ & $\begin{array}{l}13 \text { female runners } \\
\text { with amenorrhoea } \\
\text { (ages 23-34) }\end{array}$ & $\begin{array}{l}\text { Oestrogen treated }(0.0625 \mathrm{mg} \\
\text { conjugated oestrogen }(n=6) \text { or } \\
50 \mu \mathrm{g} \text { transdermal estradiol } \\
(\mathrm{n}=2)) v \text { control }(\mathrm{n}=5) \text { for } \\
24 \text { months }\end{array}$ & $\begin{array}{l}\text { Lumbar spine, femoral } \\
\text { neck, Ward's triangle } \\
\text { DXA }\end{array}$ & $\begin{array}{l}\text { Increase in lumbar spine, femoral } \\
\text { neck BMD in oestrogen treated } \\
\text { group; NS decrease in BMD in } \\
\text { control group }\end{array}$ \\
\hline & $\begin{array}{l}\text { Rickenlund } \\
\text { et } a^{\beta 8}\end{array}$ & $\begin{array}{l}38 \text { women } \\
\text { ( } 26 \text { athletes (13 } \\
\text { eumenorrhoeic, } 13 \\
\text { oligoamenorrhoeic), } \\
12 \text { eumenorrhoeic } \\
\text { non-athletes) (ages } \\
\text { 16-35) }\end{array}$ & $\begin{array}{l}\text { Each group received } 30 \mu \mathrm{g} \\
\mathrm{EE}+150 \mu \mathrm{g} \text { levonorgestrel for } \\
10 \text { months }\end{array}$ & $\begin{array}{l}\text { Lumbar spine, total } \\
\text { body DXA before } \\
\text { and after } 10 \text { months } \\
\text { of OC use }\end{array}$ & $\begin{array}{l}\text { Increase in lumbar spine BMD in } \\
\text { oligoamenorrhoeic athletes } \\
\text { (especially those with low BMD at } \\
\text { baseline); increase leg BMD in } \\
\text { eumenorrhoeic athletes (related to } \\
\text { weight-bearing exercise?) }\end{array}$ \\
\hline
\end{tabular}

OC, Oral contraceptive; BMD, bone mineral density; RCT, randomised controlled trial; $E$, ethinyl oestradiol; DXA, dual energy $x$ ray absorptiometry; DPA, dual photon absorptiometry; SPA, single photon absorptiometry; NS, non-significant;'.

Table 8 Oligo/amenorrhoeic premenopausal women: no effect of oral contraceptives on bone mineral density

\begin{tabular}{|c|c|c|c|c|c|}
\hline Study design & Reference & No of patients & OC exposure & $\begin{array}{l}\text { Measurement of BMD/ } \\
\text { bone metabolism }\end{array}$ & Results \\
\hline RCT (level 2b) & Gibson $^{69}$ & $\begin{array}{l}34 \text { women with } \\
\text { athletic oligo/ } \\
\text { amenorrhoea }\end{array}$ & $\begin{array}{l}\text { Oestrogen treated ( } 1 \mathrm{mg} \\
\text { oestriol }+2 \mathrm{mg} \text { oestradiol, } \\
\text { days } 1-12 ; 1 \mathrm{mg} \text { oestriol }+2 \mathrm{mg} \\
\text { oestradiol }+1 \mathrm{mg} \text { norethisterone } \\
\text { acetate, days } 13-22 ; 0.5 \mathrm{mg} \\
\text { oestriol }+1 \mathrm{mg} \text { oestradiol, } \\
\text { days } 23-28)+1000 \mathrm{mg} \text { calcium } \\
\text { carbonate }(\mathrm{n}=10) \mathrm{v} 1000 \mathrm{mg} \\
\text { calcium carbonate }(\mathrm{n}=14) \\
v \text { control }(\mathrm{n}=10) \text { for } 18 \text { months }\end{array}$ & $\begin{array}{l}\text { Lumbar spine, Ward's } \\
\text { triangle, femoral neck, } \\
\text { trochanteric region DXA }\end{array}$ & $\begin{array}{l}\text { NS increase in BMD from baseline in } \\
\text { oestrogen treated group }\end{array}$ \\
\hline Cohort (level 2b) & $\begin{array}{l}\text { Gremion et } \\
a^{P^{\circ}}\end{array}$ & $\begin{array}{l}30 \text { female long } \\
\text { distance runners } \\
\text { (ages 19-37) }\end{array}$ & $\begin{array}{l}9 \text { OC users, } 10 \text { eumenorrhoeic } \\
\text { non-users, } 11 \text { oligo/ } \\
\text { amenorrhoeic non-users over } \\
12 \text { months }\end{array}$ & $\begin{array}{l}\text { Lumbar spine, proximal } \\
\text { femur, midfemoral shaft } \\
\text { DXA; osteocalcin }\end{array}$ & $\begin{array}{l}\text { No change in BMD from baseline at } \\
\text { any site in OC treated group; decrease } \\
\text { in lateral lumbar spine BMD from } \\
\text { baseline in oligo/amenorrhoeic group; } \\
\text { lower osteocalcin levels in OC treated } \\
\text { group than in other } 2 \text { groups }\end{array}$ \\
\hline
\end{tabular}

OC, Oral contraceptive; BMD, bone mineral density; RCT, randomised controlled trial; EE, ethinyl oestradiol; DXA, dual energy x ray absorptiometry; NS, nonsignificant. 
Table 9 Oligo/amenorrhoeic premenopausal women: negative effect of oral contraceptives on bone mineral density

\begin{tabular}{|c|c|c|c|c|c|}
\hline Study design & Reference & No of patients & OC exposure & $\begin{array}{l}\text { Measurement of BMD/ } \\
\text { bone metabolism }\end{array}$ & Results \\
\hline Case report & Zanker et $a^{1}$ & $\begin{array}{l}1 \text { amenorrhoeic } \\
\text { athlete (followed } \\
\text { between age } \\
24.8 \text { to } 36.9 \text { years) }\end{array}$ & $\begin{array}{l}\text { For the first } 5 \text { years, } \\
\text { used } 30 \mu \mathrm{g} \mathrm{EE}+150 \mu \mathrm{g} \\
\text { desogestrel }\end{array}$ & $\begin{array}{l}\text { Lumbar spine, proximal } \\
\text { femur DXA }\end{array}$ & $\begin{array}{l}9.8 \% \text { decrease in lumbar spine } \mathrm{BMD} \text { and } \\
12.1 \% \text { decrease in proximal femur BMD } \\
\text { during } 5 \text { years of } \mathrm{OC} \text { use }\end{array}$ \\
\hline
\end{tabular}

Table 10 Anorexic premenopausal women: positive effect of oral contraceptives on bone mineral density

\begin{tabular}{|c|c|c|c|c|c|}
\hline Study design & Reference & No of patients & OC exposure & $\begin{array}{l}\text { Measurement of BMD/ } \\
\text { bone metabolism }\end{array}$ & Results \\
\hline \multirow[t]{2}{*}{ Cross sectional } & $\begin{array}{l}\text { Seeman } \\
\text { et } a P^{2}\end{array}$ & $\begin{array}{l}117 \text { women (65 } \\
\text { with AN: } 12 \text { with } 1^{\circ} \\
\text { amenorrhoea, } 16 \\
\text { with } 2^{\circ} \text { amenorrhoea } \\
\text { taking OCs, } 37 \text { with } 2^{\circ} \\
\text { amenorrhoea not } \\
\text { taking OCs; } 52 \\
\text { healthy controls) }\end{array}$ & OC users $v$ non-users & $\begin{array}{l}\text { Lumbar spine, total body, } \\
\text { proximal femur DXA }\end{array}$ & $\begin{array}{l}\text { Higher BMD in healthy control women than } \\
\text { in women with } A N \text {; greater mean lumbar } \\
\text { spine } B M D \text { in women with } A N \text { taking } O C s \\
\text { than in women with } A N \text { not taking OCs }\end{array}$ \\
\hline & $\begin{array}{l}\text { Karlsson } \\
\text { et } a l^{3}\end{array}$ & $\begin{array}{l}366 \text { women ( } 77 \text { non-OC } \\
\text { users with } A N, 58 \text { OC } \\
\text { users with AN, } 26 \\
\text { women recovered } \\
\text { from AN; } 205 \text { healthy } \\
\text { controls) }\end{array}$ & OC users $v$ non-users & $\begin{array}{l}\text { Areal BMD by DXA, } \\
\text { volumetric BMD calculated }\end{array}$ & $\begin{array}{l}\text { Higher BMD in healthy control women than } \\
\text { in women with } A N \text {; greatest reduction in } \\
\text { BMD was in non-OC users with } A N \text {; lesser } \\
\text { reduction in } O C \text { users with } A N \text {; least } \\
\text { reduction in women recovered from AN }\end{array}$ \\
\hline
\end{tabular}

Table 11 Anorexic premenopausal women: no effect of oral contraceptives on bone mineral density

\begin{tabular}{|c|c|c|c|c|c|}
\hline Study design & Reference & No of patients & OC exposure & $\begin{array}{l}\text { Measurement of } \\
\text { BMD/bone } \\
\text { metabolism }\end{array}$ & Results \\
\hline \multirow[t]{3}{*}{$\begin{array}{l}\text { RCT } \\
\text { (level 1b) }\end{array}$} & Klibanski ef $a P^{4}$ & $\begin{array}{l}48 \text { women } \\
\text { with AN } \\
\text { (ages 16-42) }\end{array}$ & $\begin{array}{l}0.625 \mathrm{mg} \text { Premarin } / 5 \mathrm{mg} \text { Provera } \\
(\mathrm{n}=16) \vee 35 \mu \mathrm{g} \text { EE }(\mathrm{n}=6) \vee \text { control } \\
(\mathrm{n}=26) \text { for } 18 \text { months }\end{array}$ & Lumbar spine CT & $\begin{array}{l}\text { No significant changes in BMD between } \\
\text { oestrogen treated and control groups; } 4 \% \\
\text { increase in BMD in oestrogen treated } \\
\text { patients with initial ideal body weight of } \\
<70 \% \text { v } 20 \% \text { decrease in BMD in control } \\
\text { patients with initial ideal body weight of } \\
<70 \%\end{array}$ \\
\hline & Gordon et $a f^{5}$ & $\begin{array}{l}51 \text { women } \\
\text { with AN } \\
\text { (ages 14-28) }\end{array}$ & $\begin{array}{l}20 \mu \mathrm{g} \mathrm{EE}+0.1 \mathrm{mg} \text { levonorgestrel } \\
\text { v } 50 \mathrm{mg} \text { dehydroepiandrosterone } \\
\text { for } 12 \text { months }\end{array}$ & $\begin{array}{l}\text { Lumbar spine, } \\
\text { total body, total } \\
\text { hip, femoral neck, } \\
\text { trochanter DXA; } \\
\text { serum osteocalcin, } \\
\text { BSAP, urinary NTx }\end{array}$ & $\begin{array}{l}\text { NS increase in lumbar BMD in both } \\
\text { groups; decrease in urinary NTx in both } \\
\text { groups (suggesting decrease in } \\
\text { resorption) }\end{array}$ \\
\hline & Grinspoon et $a P^{6}$ & $\begin{array}{l}60 \text { women } \\
\text { with AN }\end{array}$ & $\begin{array}{l}35 \mu \mathrm{g} \mathrm{EE}+0.4 \mathrm{mg} \text { norethindrone } \\
(\mathrm{n}=15) \vee 30 \mu \mathrm{g} / \mathrm{kg} \text { rhlGF-I }(\mathrm{n}=14) \\
v 30 \mu \mathrm{g} / \mathrm{kg} \text { rhIGF- } \mathrm{l}+35 \mu \mathrm{g} \mathrm{EE}+0.4 \mathrm{mg} \\
\text { norethindrone }(\mathrm{n}=16) \vee \text { control } \\
\text { (placebo rhIGF-I, no OC) }(\mathrm{n}=15) \text { for } \\
9 \text { months }\end{array}$ & $\begin{array}{l}\text { Lumbar spine, total } \\
\text { body, distal radius, } \\
\text { total hip, femoral } \\
\text { neck DXA }\end{array}$ & $\begin{array}{l}\text { Factorial analysis: no effect of } O C \text { on BMD } \\
\text { at any site; } 4 \text {-group analysis: increase in } \\
\text { AP lumbar BMD in combined rhIGF-I+OC } \\
\text { group } v \text { baseline and } v \text { placebo; Overall: } \\
\text { OCs may augment effects of rhIGF-I on } \\
\text { BMD, but are not effective alone }\end{array}$ \\
\hline $\begin{array}{l}\text { Cohort } \\
\text { (level 2b) }\end{array}$ & Golden et $a P^{7}$ & $\begin{array}{l}50 \text { women } \\
\text { with AN } \\
\text { (ages 13-21) }\end{array}$ & 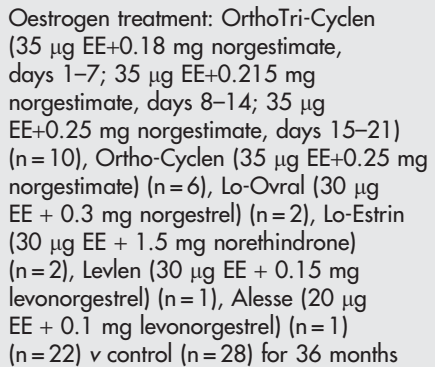 & $\begin{array}{l}\text { Lumbar spine, } \\
\text { left hip DXA }\end{array}$ & $\begin{array}{l}\text { Initial BMDs were decreased compared } \\
\text { with the young adult reference mean; no } \\
\text { significant changes in BMD from baseline } \\
\text { in either oestrogen treated or control } \\
\text { groups }\end{array}$ \\
\hline $\begin{array}{l}\text { Case series } \\
\text { (level 4) }\end{array}$ & Muñoz et $a P^{8}$ & $\begin{array}{l}38 \text { women } \\
\text { with AN (mean } \\
\text { age } 17.3 \text { years) }\end{array}$ & $\begin{array}{l}50 \mu \mathrm{g} \mathrm{EE}+0.5 \mathrm{mg} \text { norgestrel for } \\
12 \text { months }\end{array}$ & $\begin{array}{l}\text { Lumbar } \\
\text { spine DXA }\end{array}$ & No change in BMD from baseline \\
\hline
\end{tabular}

OC, Oral contraceptive; BMD, bone mineral density; RCT, randomised controlled trial; $A N$, anorexia nervosa; $E E$, ethinyl oestradiol; $C T$, computed tomography; NS, non-significant; DXA, dual energy $x$ ray absorptiometry; BSAP, bone specific alkaline phosphatase; NTx, N-telopeptides; rhIGF-I, recombinant human insulinlike growth factor $\mathrm{I}$. 
Table 12 Anorexic premenopausal women: negative effect of oral contraceptives on bone mineral density

\begin{tabular}{|c|c|c|c|c|c|}
\hline Study design & Reference & No of patients & OC exposure & $\begin{array}{l}\text { Measurement of BMD/ } \\
\text { bone metabolism }\end{array}$ & Results \\
\hline Cohort (level 2b) & Kreipe et $a f^{9}$ & $\begin{array}{l}4 \text { women with } \\
\text { AN (ages 17-28) }\end{array}$ & $\begin{array}{l}\text { Oestrogen + progestin } \\
\text { replacement }(n=2) v \text { control } \\
(n=2) \text { for } 6 \text { months }\end{array}$ & Lumbar spine DXA & $\begin{array}{l}1.9 \% \text { decrease in BMD in } \\
\text { oestrogen-progestin treated } \\
\text { group } v 1.3 \% \text { increase in BMD } \\
\text { in control group }\end{array}$ \\
\hline
\end{tabular}

Table 13 Perimenopausal women: positive effect of oral contraceptives on bone mineral density

\begin{tabular}{|c|c|c|c|c|c|}
\hline Study design & Reference & No of patients & OC exposure & $\begin{array}{l}\text { Measurement of BMD/ } \\
\text { bone metabolism }\end{array}$ & Results \\
\hline RCT (level 1b) & Volpe et $a^{\beta 0}$ & $\begin{array}{l}17 \text { perimenopausal } \\
\text { women } \\
\text { (ages } 46-53 \text { ) }\end{array}$ & $\begin{array}{l}\text { OC treated }(n=8) v \text { control } \\
(n=9) \text { for } 36 \text { months }\end{array}$ & Spine DXA & $\begin{array}{l}\text { NS increase in BMD in OC } \\
\text { users, decrease in BMD in non- } \\
\text { users }\end{array}$ \\
\hline \multirow[t]{4}{*}{$\begin{array}{l}\text { Cohort } \\
\text { (level } 2 b^{81}{ }^{83} 84 \\
\text { evel } 4^{82} \text { ) }\end{array}$} & Shargil ${ }^{81}$ & $\begin{array}{l}200 \text { perimenopausal } \\
\text { women } \\
\text { (ages } 41-49 \text { ) }\end{array}$ & $\begin{array}{l}\text { Triphasic OC ( } 30 \mu \mathrm{g} E+0.05 \mathrm{mg} \\
\text { levonorgestrel } \times 6,40 \mu \mathrm{gEE}+ \\
0.075 \mathrm{mg} \text { levonorgestrel } \times 5,30 \mu \mathrm{g} \\
\mathrm{EE}+0.125 \mathrm{mg} \text { levonorgestrel } \times 10) \\
(\mathrm{n}=100) \vee \text { control }(\mathrm{n}=100) \text { for } \\
36 \text { months }\end{array}$ & $\begin{array}{l}\text { Lumbar spine, hand bone } \\
\text { mass } x \text { ray/CT }\end{array}$ & $\begin{array}{l}\text { No change in OC users } v 6 \% \\
\text { decrease in BMD in controls }\end{array}$ \\
\hline & $\begin{array}{l}\text { Gambacciani } \\
\text { et } a^{\beta^{2}}\end{array}$ & $\begin{array}{l}32 \text { perimenopausal } \\
\text { oligomenorrhoeic } \\
\text { women }\end{array}$ & $\begin{array}{l}30 \mu \mathrm{gEE}+75 \mu \mathrm{g} \text { gestodene } \\
(\mathrm{n}=16) \vee 500 \mathrm{mg} \mathrm{Ca}^{2+}(\mathrm{n}=16) \\
\text { for } 24 \text { months }\end{array}$ & Radius DPA & Increase $B M D$ with $O C$ use \\
\hline & $\begin{array}{l}\text { Gambacciani } \\
\text { et } a^{\beta^{3}}\end{array}$ & $\begin{array}{l}90 \text { perimenopausal } \\
\text { ( } 27 \text { eumenorrhoeic, } \\
54 \text { oligomenorrhoeic) } \\
\text { women }\end{array}$ & $\begin{array}{l}20 \mu \mathrm{gEE}+0.15 \mathrm{mg} \text { desogestrel } \\
(\mathrm{n}=27) v 500 \mathrm{mg} \mathrm{Ca}^{2+}(\mathrm{n}=27) \\
\text { for } 24 \text { months }\end{array}$ & Lumbar spine DXA & $\begin{array}{l}\text { Increase in BMD with OC use } v \\
\text { decrease BMD with calcium }\end{array}$ \\
\hline & $\begin{array}{l}\text { Gambacciani } \\
\text { et } \boldsymbol{\beta}^{\beta 4}\end{array}$ & $\begin{array}{l}55 \text { perimenopausal } \\
\text { (18 eumenorrhoeic, } \\
37 \text { oligomenorrhoeic) } \\
\text { women }\end{array}$ & $\begin{array}{l}20 \mu \mathrm{g} \mathrm{EE}+0.15 \mathrm{mg} \text { desogestrel } v \\
500 \mathrm{mg} \mathrm{Ca}^{2+} \text { for } 24 \text { months }\end{array}$ & $\begin{array}{l}\text { Femoral neck, Ward's } \\
\text { triangle, trochanter DXA }\end{array}$ & $\begin{array}{l}\text { Increase in femoral neck BMD } \\
\text { from baseline with OC use } v \\
\text { decrease in femoral neck, } \\
\text { Ward's triangle, trochanter } \\
\text { BMD from baseline with } \\
\text { calcium }\end{array}$ \\
\hline \multirow[t]{3}{*}{ Cross sectional } & $\begin{array}{l}\text { Enzelsberger } \\
\text { et } a^{\beta 5}\end{array}$ & $\begin{array}{l}200 \text { perimenopausal } \\
\text { women }\end{array}$ & $\begin{array}{l}>10 \text { years OC use }(n=30) v \\
2-9 \text { years OC use }(n=50) v \\
\text { never use }(n=120)\end{array}$ & Forearm SPA & $\begin{array}{l}\text { OC use for }>10 \text { years } \\
\text { associated with increase in } \\
\text { BMD }\end{array}$ \\
\hline & 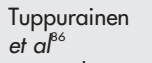 & $\begin{array}{l}3222 \text { perimenopausal } \\
\text { women }\end{array}$ & & $\begin{array}{l}\text { Lumbar spine, femoral neck } \\
\text { DXA }\end{array}$ & $\begin{array}{l}\text { Ever } O C \text { users had increase } \\
\text { spinal } B M D v \text { never users }\end{array}$ \\
\hline & $\begin{array}{l}\text { Masaryk } \\
\text { et } a^{\beta 7}\end{array}$ & $\begin{array}{l}2038 \text { women } \\
\text { (98 peri-, } 1940 \\
\text { post-menopausal) }\end{array}$ & $18.3 \%$ ever OC use & Lumbar spine, hip DXA & $\begin{array}{l}\text { Ever } O C \text { users had increase in } \\
\text { spinal } B M D \vee \text { never users }\end{array}$ \\
\hline
\end{tabular}

$\mathrm{OC}$, Oral contraceptive; BMD, bone mineral density; RCT, randomised controlled trial; DXA, dual energy $x$ ray absorptiometry; NS, non-significant; $E E$, ethinyl oestradiol; CT, computed tomography; DPA, dual photon absorptiometry; SPA, single photon absorptiometry.

Table 14 Perimenopausal women studies: no effect of oral contraceptives on bone mineral density

\begin{tabular}{|c|c|c|c|c|c|}
\hline Study design & Reference & No of patients & OC exposure & $\begin{array}{l}\text { Measurement of BMD/ } \\
\text { bone metabolism }\end{array}$ & Results \\
\hline \multirow[t]{2}{*}{ Cross sectional } & Fortney et $a f^{\beta 8}$ & $\begin{array}{l}352 \text { perimenopausal } \\
\text { women (ages 40-54) }\end{array}$ & $\begin{array}{l}\text { Ever OC users }(n=260) \\
v \text { never users }(n=92)\end{array}$ & Lumbar spine, radius DPA & $\begin{array}{l}\text { NS increase in spinal BMD in } \\
O C \text { users of longer duration } \\
\text { and more recent use }\end{array}$ \\
\hline & Beksinska et $a^{\beta 9}$ & $\begin{array}{l}496 \text { perimenopausal } \\
\text { women (ages } 40-49 \text { ) }\end{array}$ & $\begin{array}{l}\text { OC users }(30-40 \mu \mathrm{g} \mathrm{EE}) \\
(n=106) v \text { DMPA }(n=127) \\
v \text { NET-EN }(n=102) \text { (all for } \geqslant 1 \text { year) } \\
v \text { control }(n=101)\end{array}$ & $\begin{array}{l}\text { Distal radius, midshaft } \\
\text { ulna DXA }\end{array}$ & $\begin{array}{l}\text { No significant difference in } \\
\text { BMD between any of the } \\
\text { groups }\end{array}$ \\
\hline $\begin{array}{l}\text { Case series } \\
\text { (level 4) }\end{array}$ & Volpe et $a^{p o}$ & $\begin{array}{l}37 \text { perimenopausal } \\
\text { women (ages } 45-48 \text { ) }\end{array}$ & $\begin{array}{l}20 \mu \mathrm{g} \mathrm{EE}+150 \mu \mathrm{g} \text { desogestrel for } \\
24 \text { months }\end{array}$ & Lumbar spine DPA & $\begin{array}{l}\text { NS increase in BMD } \\
\text { (increase } 8 \% \text { ) }\end{array}$ \\
\hline
\end{tabular}


Table 15 Biochemical evidence: positive effect of oral contraceptives on bone metabolism

\begin{tabular}{|c|c|c|c|c|c|}
\hline Study design & Reference & No of patients & OC exposure & $\begin{array}{l}\text { Measurement of } \\
\text { bone metabolism }\end{array}$ & Results \\
\hline $\begin{array}{l}\text { Oligo/amenorrhoeic } \\
\text { RCT (level 1b) }\end{array}$ & $\begin{array}{l}\text { Grinspoon } \\
\text { et } a p^{p}\end{array}$ & $\begin{array}{l}45 \text { women with } \\
\text { hypothalamic } \\
\text { amenorrhoea } \\
\text { (ages 18-40) }\end{array}$ & $\begin{array}{l}\text { OC group ( } 35 \mu \mathrm{g} \mathrm{EE}+0.18 \mathrm{mg} \\
\text { norgestimate, days } 1-7 ; 35 \mu \mathrm{g} \\
\mathrm{EE}+0.215 \mathrm{mg} \text { norgestimate, } \\
\text { days } 8-14 ; 35 \mu \mathrm{g} E+0.25 \mathrm{mg} \\
\text { norgestimate, days } 15-21 \text { ) } \\
\text { ( } \mathrm{n}=25 \text { ) } v \text { placebo }(\mathrm{n}=20 \text { ) for } \\
3 \text { months }\end{array}$ & NTx, D-PYR & $\begin{array}{l}\text { Decrease in NTx and D-PYR in OC treated } \\
\text { group (therefore decreased resorption) }\end{array}$ \\
\hline \multicolumn{6}{|c|}{ Healthy premenopausal } \\
\hline RCT (level 1b) & Pinter et $a P^{3}$ & $\begin{array}{l}41 \text { women } \\
\text { (ages 20-27) }\end{array}$ & $\begin{array}{l}30 \mu \mathrm{gEE}+150 \mu \mathrm{g} \\
\text { levonorgestrel }(n=21) \\
v \text { control }(n=20) \text { for } 3 \text { months }\end{array}$ & $\begin{array}{l}\text { Serum BSAP and } \\
\text { osteocalcin, urinary } \\
\text { D-PYR }\end{array}$ & $\begin{array}{l}\text { OC treated: } \mathrm{BB} \text { genotype, decrease in } \\
\text { osteocalcin; in Bb genotype, decrease in BSAP } \\
\text { and osteocalcin; bb genotype, no change. } \\
\text { Control: no changes in any genotype }\end{array}$ \\
\hline \multirow[t]{2}{*}{ Cohort (level 2b) } & Paoletti et al ${ }^{p 4}$ & $\begin{array}{l}30 \text { women } \\
\text { (ages } 22-30 \text { ) }\end{array}$ & $\begin{array}{l}20 \mu \mathrm{g} \mathrm{EE}+75 \mu \mathrm{g} \text { gestodene } \\
(\mathrm{n}=10) \vee 30 \mu \mathrm{g} \mathrm{EE}+75 \mu \mathrm{g} \\
\text { gestodene }(\mathrm{n}=10) \vee \text { control } \\
(\mathrm{n}=10) \text { for } 12 \text { months }\end{array}$ & Urinary PYR, D-PYR & $\begin{array}{l}\text { Decrease in PYR, D-PYR in OC-treated groups } \\
\text { (suggesting decreased resorption) }\end{array}$ \\
\hline & Kitai et $a P^{5}$ & $\begin{array}{l}30 \text { women } \\
\text { (mean age } \\
23.7 \text { years) }\end{array}$ & OC users $v$ non-users & $\begin{array}{l}\text { Urinary } \mathrm{Ca}^{2+} / \mathrm{Cr} \\
\text { ratio }\end{array}$ & $\begin{array}{l}\text { Decrease in } \mathrm{Ca}^{2+} / \mathrm{Cr} \text { with } \mathrm{OC} \text { use (suggesting } \\
\text { decreased resorption); effect more pronounced } \\
\text { in non-smokers }\end{array}$ \\
\hline
\end{tabular}

OC, Oral contraceptive; RCT, randomised controlled trial; EE, ethinyl oestradiol; NTx, N-telopeptides; D-PYR, deoxypyridinoline; BSAP, bone specific alkaline phosphatase; PYR, pyridinoline; Cr, creatinine.

growth factor I (IGF-I), OCs plus IGF-I, or placebo plus IGF-I on BMD at several skeletal sites. No effect of OCs on BMD was detected at any site by factorial analysis, but by four group analysis it was found that, despite being ineffective alone, OCs may augment the effects of IGF-I on BMD in anorexic women. ${ }^{76}$ No RCT showed a negative effect of OC treatment on BMD.

\section{Perimenopausal women}

Eleven studies on perimenopausal women were reviewed. Eight (one RCT, ${ }^{80}$ four cohort, ${ }^{81-84}$ three cross sectional ${ }^{85-87}$ ) supported a positive effect, whereas three (two cross sectional, $^{88}{ }^{89}$ one case series ${ }^{90}$ ) showed no effect. Volpe et $a l^{80}$ showed a non-significant increase in spinal BMD in the OC treated group compared with a significant decrease in $\mathrm{BMD}$ in the control group. No study showed a negative effect of OC treatment on BMD.

\section{DISCUSSION}

This review critically examines current literature to determine the effect of OCs (and other hormone treatment) on BMD in four groups: healthy premenopausal, "hypothalamic" oligo/ amenorrhoeic premenopausal, anorexic premenopausal, and perimenopausal women. Because of the number and diversity of the studies, it was not possible to perform a formal metaanalysis of the results. However, the type of evidence, based on study type and including subject numbers, is summarised below.

There is good evidence supporting a positive effect of OCs on BMD in perimenopausal women. Of 11 studies found, eight (with a combined total of 5854 subjects) showed a positive effect, including one RCT (with 17 subjects). Three studies (of 885 women) did not find any effect. No study showed a negative effect.

There is also fair evidence supporting a positive effect of OCs on BMD in oligo/amenorrhoeic premenopausal women. Of 10 studies, seven (with a total of 379 subjects) showed a positive effect, including two RCTs in a total of 88 women. Although another RCT of 34 women reported no effect, there was still a non-significant trend towards increased BMD in the OC group in this study. In addition, a RCT of 45 women examining the effect of OCs on bone metabolism showed decreased markers of bone resorption in the OC treated group, compared with placebo, supporting a beneficial effect of OCs in this group ${ }^{91}$ (table 15). Only one case report showed a negative effect.

There is limited evidence supporting a positive effect of OCs on BMD in anorexic premenopausal women. Of eight studies, two cross sectional ones of 483 women found a positive effect. Five studies (with 247 total subjects) showed no effect. However, it appears that body weight at initiation of OC treatment may play a role in determining the effect of OCs on BMD. ${ }^{74}$ Thus, calculation of body weight, as a percentage of ideal, may be an important step in deciding whether to treat anorexic patients with OCs. This evidence may not be helpful in deciding treatment for women with the female athlete triad though, as anorexics are quite distinct in their hormonal condition and state of activity. SundgotBorgen \& Torstveit $^{92}$ reported that a higher percentage of Norwegian elite athletes met the criteria for subclinical eating disorders-that is, athletic amenorrhoea or "eating disorders not otherwise specified" - than for clinical eating disorders (anorexia or bulimia nervosa). Women with clinical eating disorders are more sedentary than women with the female athlete triad syndrome, and oestrogen deficiency appears to play less of a role, and IGF-I deficiency more of a role, in decreased BMD in women with clinical eating disorders than in those with the syndrome. ${ }^{76}$

There is limited evidence supporting a positive effect of OCs on BMD in healthy premenopausal women. Of 46 studies, 29 showed no effect, including all of the RCTs. However, one RCT ${ }^{29}$ showed a non-significant trend towards increased BMD, and three RCTs ${ }^{27-29}$ showed decreased concentrations of bone resorption markers in the OC group. Likewise, one RCT $^{93}$ and two cohort studies ${ }^{94}{ }^{95}$ examining the effect of OCs on bone metabolism also suggested similar beneficial results (table 15). A total of seven studies (cohort and cross sectional) of 1361 women suggested a negative effect of OCs on BMD. This is somewhat worrisome, and a variety of potential explanations were given.

Interestingly, there are also data from three studies showing that a combination of exercise and OC use in healthy premenopausal women may have a negative effect on BMD. Postulated reasons for the negative interaction between exercise and OC use are: inadequate bone mineralisation because of nutritional calcium deficiency, ${ }^{56} 57$ suppression of endogenous pituitary releasing hormone, oestrogen, and progesterone peaks with resultant alteration 
of the bone mechanostat, ${ }^{59}$ and the differential effects of different progestins on BMD. ${ }^{59}$

According to the Oxford Centre for Evidence-Based Medicine levels of evidence, ${ }^{15}$ the strongest level of evidence (la) is derived from a systematic review with homogeneity of RCTs. The next best level (lb) is from individual RCTs, with evidence from other study designs carrying less weight. In this review, focus was placed on the RCTs, with supporting evidence from other study types. All of the RCTs included had methodological limitations. In three of the RCTs, subjects were asked whether they desired contraception or not. Those that desired contraception were randomised to one of several treatment groups, and those who did not choose contraception served as the controls, necessitating the concern of self selection bias. ${ }^{27} 2963$ Three other studies compared the effect of different types/doses of OCs on BMD, but did not include a non-treatment control group for comparison. ${ }^{26} 2875$ Five studies had non-treatment control groups ${ }^{62} 69747680$ but only one was placebo controlled. ${ }^{62}$ Only one study was double blinded, ${ }^{28}$ but two other studies were single blinded. ${ }^{27} 62$ Reported reasons for not including a placebo control and for not blinding subjects were: the expected bone loss if a placebo control was used, ${ }^{75}$ and the expected withdrawal bleeding in subjects who were initially amenorrhoeic taking OCs. ${ }^{76}$ The duration of the RCTs ranged from nine months ${ }^{76}$ to three years. ${ }^{31}{ }^{80}$ The follow up rate was good, being $<80 \%$ in only two studies. ${ }^{28} 69$

The cohort studies included in this review were generally of good quality. In all of them, BMD was measured in the same way in both the OC exposed and non-exposed groups, and confounding variables were identified and accounted for. Further, the groups were similar, ${ }^{17} 1830-32343555-576467707981-84$ and the follow up rate was $>80 \%{ }^{16} 1830-32356467707779818384$ in most of the studies. However, in several of the studies, follow up was $<80 \%$, ${ }^{17} 333455-57656682$ and the groups differed in factors potentially contributing to selection bias. ${ }^{33656677}$

Many of the studies reviewed were cross sectional. ${ }^{19-25}{ }^{45-}$ 53 59-61 72 73 85-89 In addition, three case series ${ }^{54} 7890$ and one case report $^{71}$ were also reviewed. Evidence from these types of study is weaker, as confounding variables are less likely to have been controlled for, and the results may be more subject to selection and recall bias. Cross sectional studies and case reports are not specifically classified under the Oxford Centre for Evidence-Based Medicine levels of evidence; however, it was felt that they could provide useful evidence that should be included in this review.

A review by Kuohung et $a l^{96}$ evaluated 13 studies examining the effect of low dose OCs-that is, 20-40 $\mu \mathrm{g}$ ethinyl oestradiol—on BMD in women of all ages, including postmenopausal women. Their results suggested that there was fair evidence supporting a favourable effect of OC use on BMD. ${ }^{96}$ However, in premenopausal and perimenopausal women, there have been mixed results. Previous reviews have attributed these divergent results to differences in study design, ${ }^{47}{ }^{98}$ inadequate sample sizes, ${ }^{497}$ and heterogeneity in study populations, because of the many confounders affect-

\section{What is already known on this topic}

- To date, there have been mixed results (either positive or no effect) in studies examining the effect of oral contraceptives and other hormone therapy on bone density in healthy premenopausal and perimenopausal women

- Previous reviews have not taken into account health or menstrual status ing $\mathrm{BMD}^{2{ }^{2}}$ such as genetics (race), lifestyle (smoking, alcohol, nutrition, exercise), and hormonal (menstrual history, age at menarche, parity, breast feeding) factors. There was a wide diversity in study populations examined among the papers reviewed, but we attempted to define more homogeneous populations by classifying studies into four groups according to health, menstrual status, and reproductive age (premenopausal or perimenopausal). However, an important distinction between reproductive age and skeletal age should be noted. As the average age of menopause ranges from 40 to 58 years, ${ }^{99}$ a woman classified as "premenopausal" can be anywhere from age 40 and below, and thus may be either skeletally immature or mature. Recker et al ${ }^{16}$ found that women do not reach skeletal maturity, as reflected by peak bone mass, until around 30 years of age. As skeletal maturity was not an inclusion criterion in any of the studies reviewed, it is unclear whether the subjects had attained peak bone mass or not. This heterogeneity in skeletal maturity may be partly responsible for the variability in results, especially in the cohort and cross sectional studies in healthy premenopausal women, where the evidence seemed to be split between positive effect and no effect. Interestingly, an RCT conducted in skeletally immature cynomolgus monkeys showed that OC treatment actually inhibited net bone accretion and/or growth by reducing bone metabolism, ${ }^{100}$ whereas no RCT in humans has yet shown a negative effect of OCs on BMD. Thus there is the potential that the effect of OC treatment on BMD may be, in part, dependent on skeletal (rather than reproductive) maturity.

Other factors affecting the results include the method and anatomical site of BMD measurement. Among the reviewed studies, there were seven different methods used: ${ }^{125}$ I photon absorptiometry, ${ }^{19} 39$ single photon absorptiometry, ${ }^{16} 213057648588 \times$ ray/computed tomography, ${ }^{81}$ quantitative computed tomography, ${ }^{28} 41$ dual photon absorptiometry, ${ }^{16} 2021304042515464828890$ single $X$ ray absorptiometry, ${ }^{47} 4950$ and DXA. ${ }^{17} 1822-2729$ 31-38 43-46 $485255-63656889$ There were six different anatomical sites of BMD measurement: lumbar spine, ${ }^{16} 17$ 20-25 27-31 $3335384042444548515457-63647274-$ $818386-90$ hip (femoral neck, trochanter, Ward's triangle), ${ }^{22-}$ $253233353840424445485156-62666769$ 70-72 75-77 84 8687 hand, ${ }^{81}$ heel, ${ }^{37}$ radius, ${ }^{16} 19212330454749-547682858889$ and total body..$^{1623-}$ 2533354546485257626872737576 This is important because the type of bone varies between anatomical site-for example, vertebral bodies are primarily trabecular, whereas the femur is predominantly cortical, ${ }^{62}$ - and each method allows more accurate measurement of different types of bone-for example, DXA for trabecular, single photon absorptiometry for cortical. ${ }^{96}$ Furthermore, trabecular bone is more active than cortical; thus the effects of oestrogen may be more readily apparent in trabecular bone. ${ }^{4}$ Variations in location and method of BMD measurement may also account for previous discordant findings.

The type, dose, and formulation of OC used also differed between the studies reviewed. In two studies, mestranol was used, $^{19}{ }^{32}$ whereas in the rest, various doses of ethinyl

What this study adds

- This study reviews the evidence in premenopausal and perimenopausal women, including all study types (randomised controlled trials, as well as all other types)

- The studies are stratified according to health, menstrual status, and reproductive age, in order to more clearly define effects of oral contraceptives and other hormone therapy on bone mineral density in each group 
oestradiol were used $\left(10 \mu g^{65} 20 \mu g^{38} 54555863657577838490\right.$ $30 \mu g^{17262931363745636566687177818289}$

$35 \mu \mathrm{g},{ }^{17} 2636486265747677 \leqslant 50 \mu \mathrm{g},{ }^{47} 565781 \quad 50-100 \mu \mathrm{g},{ }^{19} 45646578$ or unknown/unspecified doses, ${ }^{16} 18$ 20-25 $3032-34$ 49$515960707273798085-88$ ) and in combination with six different progestins or other hormones (levonorgestrel, ${ }^{28} 38485668757781$ norgestrel, ${ }^{2077} 78$ norgestimate, ${ }^{77}$ norethindrone, ${ }^{17} 3648627677$ gestodene, $^{27} 2982$ desogestrel $^{17} 2631365455636671838488$ cyproterone acetate, ${ }^{2664}$ or drospirenone $\left.{ }^{29}{ }^{37}\right)$. A study on postmenopausal women examining the effect of oestrogen dose on bone loss has suggested a dose-response effect: at $<15 \mu \mathrm{g}$ ethinyl oestradiol, net bone loss occurs, and at $>25 \mu \mathrm{g}$ ethinyl oestradiol, net bone gain occurs, but between 15 and $25 \mu \mathrm{g}$ ethinyl oestradiol, neither bone gain nor loss occurs. ${ }^{101}$ If this dose-response effect holds true in premenopausal and perimenopausal women, the doses used in some of the studies may have been insufficient to show any effect on BMD. In addition, different progestins vary in their effects on bone. $^{97102103}$ For example, one study showed that a portion of norethindrone is converted into ethinyl oestradiol in the body, resulting in potential bone-sparing properties. ${ }^{104}$

The definition of OC exposure also differed greatly in the cohort and cross sectional studies. Some used the "non-user" $v$ "user" distinction, ${ }^{18}$ 32-34 4145 51-53 55-57 64-67 72737779 81-84 some used "ever" $v$ "never", 20-23404344476088 and others further subdivided "ever" users into "current" and "past" users. ${ }^{1625} 3050$ Still others used specific time periods to define OC users-for example, $>2$ months, ${ }^{39} \geqslant 6$ months and still at the age of $22,{ }^{35} \geqslant 2$ years, ${ }^{49} \geqslant 4$ years, ${ }^{70}$ never $/ 2-9$ years $/>10$ years, ${ }^{85}$ or $>3$ years if $<22$ years old or $>50 \%$ of the time after menarche if $>22$ years old ${ }^{61}$ - yet these time periods seemed arbitrary, as no reasons for their selection were given. Cobb et $a l^{24}$ have suggested the concept of "cumulative oestrogen exposure" as a quantitative method of defining OC exposure, derived by multiplying the oestrogen dose per month by the total number of months that OCs were used. Use of this quantitative method in the future may make comparison between studies easier.

Clearly, a number of confounding variables influence the effect of OCs on BMD, which may contribute to the divergent results in the literature.

\section{CONCLUSION}

There is good evidence for a positive effect of OCs on BMD in perimenopausal women, and fair evidence in "hypothalamic" oligo/amenorrhoeic premenopausal women. However, there is limited evidence in anorexic and healthy premenopausal women for any positive effect. Further RCTs should be carried out to confirm these results. Ideally, any future studies would also take into account skeletal maturity, as well as reproductive maturity. In addition, studies of women with menstrual dysfunction should use consistent definitions of eumenorrhoea, oligomenorrhoea, and amenorrhoea.

Of significance to the female athlete is the combined effect of OCs and exercise on BMD, but to date there is a lack of evidence in this area. Ultimately, the decision to prescribe OCs to support BMD in the female athlete should be made on an individual basis, taking into account lifestyle and hormonal factors. Current literature does not show any evidence of a negative effect of OC use on BMD in women. OC use may have a favourable effect on BMD, especially in premenopausal women with athletic oligo/amenorrhoea. In these women, baseline BMD has been shown to be significantly lower than that in healthy controls; therefore the decision to treat is clinically more important. Hence, in oligo/amenorrhoeic athletes, the best therapeutic option to support BMD in those desiring contraception, or in those athletes in whom other conservative measures have not resulted in return of normal ovulatory menses in a reasonable amount of time, may be OCs. The "ideal" formulation(s) and duration of treatment remain to be determined by further longitudinal and prospective RCTs.

\section{Authors' affiliations}

S L Liv, Queen's University, Kingston, Ontario, Canada

C M Lebrun, Fowler Kennedy Sport Medicine Clinic, University of

Western Ontario, London, Ontario, Canada

Competing interests: none declared

\section{REFERENCES}

1 Wilkins K, Johansen $\mathrm{H}$, Beaudet MP, et al. Oral contraceptive use. Health Rep 2001;11:25-37.

2 Bennell K, White S, Crossley K. The oral contraceptive pill: a revolution for sportswomen? Br J Sports Med 1999;33:231-8

3 Jensen JT, Speroff L. Health benefits of oral contraceptives. Obstet Gynecol Clin North Am 2000;27:705-21.

4 Corson SL. Oral contraceptives for the prevention of osteoporosis. J Reprod Med 1993;38:1015-20.

5 Yeager KK, Agostini R, Nattiv A, et al. The female athlete triad: disordered eating, amenorrhea, osteoporosis. Med Sci Sports Exerc 1993;25:775-7.

6 WHO Study Group. Assessment of fracture risk and its application to screening for postmenopausal osteoporosis. World Health Organ Tech Rep Ser 1994;843:1-129.

7 Writing Group for the ISCD Position Development conference. Diagnosis of osteoporosis in men, premenopausal women, and children. J Clin Densitom 2004;7:17-26.

8 Khan KM, Liu-Ambrose T, Sran MM, et al. New criteria for female athlete triad syndrome? As osteoporosis is rare, should osteopenia be among the criteria for defining the female athlete triad syndrome? Br J Sports Med 2002;36:10-13.

9 Kazis K, Iglesias E. The female athlete triad. Adolesc Med 2003;14:87-95.

10 Riggs BL, Khosla S, Melton $\sqcup$ 3rd. ex steroids and the construction and conservation of the adult skeleton. Endocr Rev 2002;23:279-302.

11 Eriksen EF, Colvard DS, Berg NJ, et al. Evidence of estrogen receptors in normal human osteoblast-like cells. Science 1988;241:84-6.

12 Oursler MJ, Osdoby P, Pyfferoen J, et al. Avian osteoclasts as estrogen target cells. Proc Nat Acad Sci USA 1991;88:6613-17.

13 Balasch J. Sex steroids and bone: current perspectives. Hum Reprod Update 2003;9:207-22.

14 Frost HM. The role of changes in mechanical usage set points in the pathogenesis of osteoporosis. J Bone Miner Res 1992;7:253-61.

15 Phillips B, Ball C, Sackett D, et al. Oxford Centre for Evidence-Based Medicine Levels of Evidence (May 2001). http://www.cebm.net/ levels_of_evidence.asp (accessed 20 Oct 2005).

16 Recker RR, Davies KM, Hinders SM, et al. Bone gain in young adult women. JAMA 1992;268:2403-8.

17 Berenson AB, Radecki CM, Grady JJ, et al. A prospective, controlled study of the effects of hormonal contraception on bone mineral density. Obstet Gynecol $2001 ; 98: 576-82$

18 Elgán C, Samsioe G, Dykes AK. Influence of smoking and oral contraceptives on bone mineral density and bone remodeling in young women: a 2-year study. Contraception 2003;67:439-47.

19 Goldsmith NF, Johnston JO. Bone mineral effects of oral contraceptives, pregnancy and lactation. J Bone Joint Surg 1975;57:657-68.

20 Lindsay R, Tohme J, Kanders B. The effect of oral contraceptive use on vertebral bone mass in pre- and post-menopausal women. Contraception 1986;34:333-40.

21 Kleerekoper $M$, Brienza RS, Schultz LR, et al. Oral contraceptive use may protect against low bone mass: Henry Ford Hospital Osteoporosis Cooperative Research Group. Arch Intern Med 1991;151:1971.

22 Laitinen K, Valimaki M, Keto P. Bone mineral density measured by dualenergy X-ray absorptiometry in healthy Finnish women. Calcif Tissue Int 1991;48:224-31.

23 Pasco JA, Kotowicz MA, Henry MJ, et al. Oral contraceptives and bone mineral density: a population-based study. Am J Obstet Gynecol 2000; 182:265-9.

24 Cobb KL, Kelsey JL, Sidney S, et al. Oral contraceptives and bone mineral density in white and black women in CARDIA. Osteoporos Int 2002; 13:893-900

25 Wallace LS, Ballard JE. Lifetime physical activity and calcium intake related to bone density in young women. J Womens Health Gend Based Med 2002;11:389-98.

26 Castelo-Branco C, Martinez de Osaba MJ, Pons R, et al. Effects on bone mass of two oral contraceptives containing ethinylestradiol and cyproterone acetate or desogestrel: results of a 2-year follow-up. Eur J Contracept Reprod Health Care 1998:3:79-84.

27 Nappi C, Di Spiezio Sardo A, Acunzo G, et al. Effects of a low-dose and ultra-low-dose combined oral contraceptive use on bone turnover and bone mineral density in young fertile women: a prospective controlled randomized study. Contraception 2003;67:355-9.

28 Endrikat J, Mih E, Dusterberg B, et al. A 3 year double-blind randomized controlled study on the influence of 2 oral contraceptives containing either $20 \mu \mathrm{g}$ or $30 \mu \mathrm{g}$ ethinylestradiol in combination with levonorgestrel on bone mineral density. Contraception 2004;69:179-87. 
29 Nappi C, Di Spiezio Sardo A, Greco E, et al. Effects of an oral contraceptive containing drospirenone on bone turnover and bone mineral density. Obstet Gynecol 2005; 105:53-60.

30 Mazess RB, Barden HS. Bone density in premenopausal women: effects of age, dietary intake, physical activity, smoking, and birth control pills. Am J Clin Nutr 1991;53:132-42.

31 Cromer BA, Blair JM, Mahan JD, et al. A prospective comparison of bone density in adolescent girls receiving depot medroxyprogesterone acetate, levonorgestrel, or oral contraceptives. J Pediatr 1996;129:671-6.

32 Lloyd T, Taylor DS, Lin HM, et al. Oral contraceptive use by teenage women does not affect peak bone mass: a longitudinal study. Fertil Steril 2000;74:734-8.

33 Reed SD, Scholes D, LaCroix AZ, et al. Longitudinal changes in bone density in relation to oral contraceptive use. Contraception 2003;68:177-82.

34 Lara-Torre E, Edwards CP, Perlman S, et al. Bone mineral density in adolescent females using depot medroxyprogesterone acetate. J Pediatr Adolesc Gynecol 2004;17:17-21.

35 Lloyd T, Petit MA, Lin HM, et al. Lifestyle factors and the development of bone mass and bone strength in young women. J Pediatr 2004;144:776-82.

36 Berenson AB, Breitkopf CR, Grady JJ, et al. Effects of hormonal contraception on bone mineral density after 24 months of use. Obstet Gynecol 2004; 103:899-906.

37 Paoletti AM, Orru M, Lello S, et al. Short-term variations in bone remodeling markers of an oral contraception formulation containing $3 \mathrm{mg}$ of drospirenone plus 30 microg of ethinyl estradiol: observational study in young postadolescent women. Contraception 2004;70:293-8.

38 Rome E, Ziegler J, Secic $M$, et al. Bone biochemical markers in adolescent girls using either depot medroxyprogesterone acetate or an oral contraceptive. J Pediatr Adolesc Gynecol 2004;17:373-7.

39 Sowers M, Wallace RB, Lenke JH. Correlates of forearm bone mass among women during maximal bone mineralization. Prev Med 1985;14:585-96.

40 Hreshchyshyn MM, Hopkins A, Zylstra S, et al. Associations of parity, breast-feeding, and birth control pills with lumbar spine and femoral neck bone densities. Am J Obstet Gynecol 1988;159:318-22.

41 Lloyd T, Buchanan JR, Ursino GR, et al. Long-term oral contraceptive use does not affect trabecular bone density. Am J Obstet Gynecol 1989; 160:402-4.

42 Stevenson JC, Lees B, Devenport $M$, et al. Determinants of bone density in normal women: risk factors for future osteoporosis. BMJ 1989;298:924-8

43 Hall ML, Heavens J, Cullum ID, et al. The range of bone density in normal British women. Br J Radiol 1990;63:266-9.

44 Murphy S, Khaw KT, Compston JE. Lack of relationship between hip and spine bone mineral density and oral contraceptive use. Eur J Clin Invest 1993;23:108-11.

45 Garnero P, Sornay-Rendu E, Delmas PD. Decreased bone turnover in oral contraceptive users. Bone 1995;16:499-503.

46 Ulrich CM, Georgiou CC, Snow-Harter CM, et al. Bone mineral density in mother-daughter pairs: relations to lifetime exercise, lifetime milk consumption, and calcium supplements. Am J Clin Nutr 1996;63:72-9.

47 Petitti DB, Piaggio G, Mehta S, et al. Steroid hormone contraception and bone mineral density: a cross-sectional study in an international population. Obstet Gynecol 2000;95:736-44.

48 Ott SM, Scholes D, Lacroix AZ, et al. Effects of oral contraceptive use on bone biochemical markers in young women. J Clin Endocrinol Metab 2001;86:179-85.

49 Perrotti M, Bahamondes L, Petta C, et al. Forearm bone density in long-term users of oral combined contraceptives and depot medroxyprogesterone acetate. Fertil Steril 2001;76:469-73.

50 Hawker GA, Forsmo S, Cadarette SM, et al. Correlates of forearm bone mineral density in young Norwegian women. Am J Epidemiol 2002;156:418-27.

51 Wanichsetakul P, Kamudhamar A, Watanarunagkaut $P$, et al. Bone mineral density at various anatomic bone sites in women receiving combined oral contraceptives and depot medroxyprogesterone acetate for contraception. Contraception 2002;65:407-10.

52 Afghani A, Abbott AV, Wiswell RA, et al. Bone mineral density in Hispanic women: role of aerobic capacity, fat-free mass, and adiposity. Int J Sports Med 2004;25:384-90.

53 Meyer NL, Shaw JM, Manore MM, et al. Bone mineral density of olympiclevel female winter sport athletes. Med Sci Sports Exerc 2004;36:1594-601

54 Mais V, Fruzzetti F, Ajossa S, et al. Bone metabolism in young women taking a monophasic pill containing $20 \mathrm{mcg}$ ethinylestradiol: a prospective study. Contraception 1993;48:445-52.

55 Polatti F, Perotti F, Filippa N, et al. Bone mass and long-term monophasic oral contraceptive treatment in young women. Contraception 1995;51:221-4.

56 Burr DB, Yoshikawa T, Teegarden D, et al. Exercise and oral contraceptive use suppress the normal age-related increase in bone mass and strength of the femoral neck in women 18-31 years of age. Bone 2000;27:855-63.

57 Weaver CM, Teegarden D, Lyle RM, et al. Impact of exercise on bone health and contraindication of oral contraceptive use in young women. Med Sci Sports Exerc 2001;33:873-80.

58 Cromer BA, Stager M, Bonny A, et al. Depot medroxyprogesterone acetate, oral contraceptives and bone mineral density in a cohort of adolescent girls. $J$ Adolesc Health 2004;35:434-41

59 Hartard M, Bottermann P, Bartenstein P, et al. Effects on bone mineral density of low-dosed oral contraceptives compared to and combined with physical activity. Contraception 1997;55:87-90.

60 Prior JC, Kirkland SA, Joseph L, et al. Oral contraceptive use and bone mineral density in premenopausal women: cross-sectional, population-based data from the Canadian Multicentre Osteoporosis Study. CMAJ $2001 ; 165: 1023-9$

61 Hartard M, Kleinmond C, Kirchbichler A, et al. Age at first oral contraceptive use as a major determinant of vertebral bone mass in female endurance athletes. Bone 2004; 35:836-41.

62 Hergenroeder AC, Smith EO, Shypailo R, et al. Bone mineral changes in young women with hypothalamic amenorrhea treated with oral contraceptives, medroxyprogesterone, or placebo over 12 months. Am J Obstet Gynecol 1997;176:1017-25.

63 Castelo-Branco C, Vicente JJ, Pons F, et al. Bone mineral density in young, hypothalamic oligoamenorrheic women treated with oral contraceptives. $J$ Reprod Med 2001;46:875-9.

64 De Crée C, Lewin R, Ostyn M. Suitability of cyproterone acetate in the treatment of osteoporosis associated with athletic amenorrhea. Int J Sports Med 1988;9:187-92.

65 Gulekli B, Davies MC, Jacobs HS. Effect of treatment on established osteoporosis in young women with amenorrhea. Clin Endocrinol (Oxf) 1994;41:275-81.

66 Haenggi W, Casez JP, Birkhaeuser MH, et al. Bone mineral density in young women with long-standing amenorrhea: limited effect of hormone replacement therapy with ethinylestradiol and desogestrel. Osteoporos Int 1994:4:99-103.

67 Cumming DC. Exercise associated amenorrhea, low bone density, and estrogen replacement therapy. Arch Intern Med 1996;156:2193-5.

68 Rickenlund A, Carlstrom K, Ekblom B, et al. Effects of oral contraceptives on body composition and physical performance in female athletes. J Clin Endocrinol Metab 2004;89:4364-70.

69 Gibson JH. Treatment of reduced bone mineral density in athletic amenorrhea: a pilot study. Osteoporos Int 1999;10:284-9

70 Gremion G, Rizzoli R, Slosman D, et al. Oligo-amenorrheic long-distance runners may lose more bone in spine than in femur. Med Sci Sports Exerc $2001 ; 33: 15-21$

71 Zanker CL, Cooke CB, Truscott JG, et al. Annual changes of bone density over 12 years in an amenorrheic athlete. Med Sci Sports Exerc 2004;36:137-42.

72 Seeman E, Szmukler Gl, Formica C, et al. Osteoporosis in anorexia nervosa: the influence of peak bone density, bone loss, oral contraceptive use, and exercise. J Bone Miner Res 1992;7:1467-74.

73 Karlsson MK, Weigall SJ, Duan Y, et al. Bone size and volumetric density in women with anorexia nervosa receiving estrogen replacement therapy and in women recovering from anorexia nervosa. J Clin Endocrinol Metab 2000;85:3177-82.

74 Klibanski A, Biller BM, Schoenfeld DA, et al. The effects of estrogen administration on trabecular bone loss in young women with anorexia nervosa. J Clin Endocrinol Metab 1995;80:898-904.

75 Gordon CM, Grace E, Emans SJ, et al. Effects of oral dehydroepiandrosterone on bone density in young women with anorexia nervosa: a randomized trial. J Clin Endocrinol Metab 2002;87:4935-41.

76 Grinspoon S, Thomas L, Miller K, et al. Effects of recombinant human IGF-I and oral contraceptive administration on bone density in anorexia nervosa. $J$ Clin Endocrinol Metab 2002;87:2883-91.

77 Golden NH, Lanzkowsky L, Schebendach J, et al. The effect of estrogenprogestin treatment on bone mineral density in anorexia nervosa. J Pediatr Adolesc Gynecol 2002;15:135-43.

78 Muñoz MT, Morandé G, García-Centenera JA, et al. The effects of estrogen administration on bone mineral density in adolescents with anorexia nervosa. Eur J Endocrinol 2002;146:45-50.

79 Kreipe RE, Hicks DG, Rosier RN, et al. Preliminary findings on the effects of sex hormones on bone metabolism in anorexia nervosa. J Adolesc Health 1993; 14:319-24.

80 Volpe A, Amram A, Cagnacci A, et al. Biochemical aspects of hormonal contraception: effects on bone mineral density and metabolism. Eur J Contracept Reprod Health Care 1997;2:123-6.

81 Shargil AA. Hormone replacement therapy in perimenopausal women with a triphasic contraceptive compound: a three year prospective study. Int J Fertil 1985;30:15.

82 Gambacciani M, Spinetti A, Cappagli B, et al. Hormone replacement therapy in perimenopausal women with a low dose oral contraceptive preparation: effects on bone mineral density and metabolism. Maturitas 1994;19:125-31.

83 Gambacciani M, Spinetti A, Taponeco F, et al. Longitudinal evaluation of perimenopausal vertebral bone loss: effects of a low-dose oral contraceptive preparation on BMD and metabolism. Obstet Gynecol 1994;83:392-6.

84 Gambacciani M, Ciaponi M, Cappagli B, et al. Longitudinal evaluation of perimenopausal bone loss: effects of a low-dose oral contraceptive preparation on bone mineral density and metabolism. Osteoporos Int 2000;1 1:544-8.

85 Enzelsberger H, Metka M, Heytmanek G, et al. Influence of oral contraceptive use on bone density in climacteric women. Maturitas 1988;9:375-8.

86 Tuppurainen $M$, Kroger $H$, Saarikoski S, et al. The effect of previous oral contraceptive use on bone mineral density in perimenopausal women. Osteoporos Int 1994;4:93-8.

87 Masaryk P, Lunt $M$, Benevolenskaya L, et al. Effects of menstrual history and use of medications on bone mineral density: the EVOS Study. Calcif Tissue Int 1998;63:271-6

88 Fortney JA, Feldblum PJ, Talmage RV, et al. Bone mineral density and history of oral contraceptive use. J Reprod Med 1994;39:105-9.

89 Beksinska ME, Smit JA, Kleinschmidt I, et al. Bone mineral density in women aged $40-49$ years using depot-medroxyprogesterone acetate, 
norethisterone enanthate or combined oral contraceptives for contraception. Contraception 2005;71:170-5.

90 Volpe A, Silferi $M$, Genazzani $A D$, et al. Contraception in older women. Contraception 1993:47:229-39.

91 Grinspoon SK, Friedman AJ, Miller KK, et al. Effects of a triphasic combination oral contraceptive containing norgestimate/ethinyl estradiol on biochemical markers of bone metabolism in young women with osteopenia secondary to hypothalamic amenorrhea. J Clin Endocrinol Metab 2003;88:3651-6.

92 Sundgot-Borgen J, Torstveit MK. Prevalence of eating disorders in elite athletes is higher than in the general population. Clin J Sport Med 2004; 14:25-32.

93 Pinter B, Kocijancic A, Marc J, et al. Vitamin D receptor gene polymorphism and bone metabolism during low-dose oral contraceptive use in young women. Contraception 2003:67:33-7.

94 Paoletti AM, Orrù M, Floris S, et al. Evidence that treatment with monophasic oral contraceptive formulations containing ethinylestradiol plus gestodene reduces bone resorption in young women. Contraception 2000:61:259-63.

95 Kitai E, Blum M, Kaplan B. The bone sparing effect of oral contraceptive use in non-smoking women. Clin Exp Obstet Gynecol 1992;19:30-3.
96 Kuohung W, Borgatta L, Stubblefield P. Low-dose oral contraceptives and bone mineral density: an evidence-based analysis. Contraception 2000;61:77-82.

97 DeCherney A. Bone-sparing properties of oral contraceptives. Am J Obstet Gynecol 1996;174:15-20.

98 Gordon CM, Nelson LM. Amenorrhea and bone health in adolescents and young women. Curr Opin Obstet Gynecol 2003;15:377-84.

99 Lobo RA. Menopause. In: Goldman L, Bennett JC, eds. Cecil textbook of medicine. 21 st ed. Philadelphia, PA: WB Saunders Company, 2000:1361.

100 Register TC, Jayo MJ, Jerome CP. Oral contraceptive treatment inhibits the normal acquisition of bone mineral in skeletally immature young adult female monkeys. Osteoporos Int 1997;7:348-53.

101 Horsman A, Jones M, Francis R, et al. The effect of estrogen dose on postmenopausal bone loss. N Engl J Med 1983;309:1405-7.

102 DeCherney A. Physiologic and pharmacologic effects of estrogen and progestins on bone. J Reprod Med 1993;38:1007-14.

103 Cromer BA. Bone mineral density in adolescent and young adult women on injectable or oral contraception. Curr Opin Obstet Gynecol 2003;15:353-7.

104 Reed MJ, Ross MS, Lai LC, et al. In vivo conversion of norethisterone to ethinyl estradiol in perimenopausal women. J Steroid Biochem Med Biol 1990;37:301-3.

\section{Clinical Evidence-Call for contributors}

Clinical Evidence is a regularly updated evidence-based journal available worldwide both as a paper version and on the internet. Clinical Evidence needs to recruit a number of new contributors. Contributors are healthcare professionals or epidemiologists with experience in evidence-based medicine and the ability to write in a concise and structured way.

Areas for which we are currently seeking contributors:

- Pregnancy and childbirth

- Endocrine disorders

- Palliative care

- Tropical diseases

We are also looking for contributors for existing topics. For full details on what these topics are please visit www.clinicalevidence.com/ceweb/contribute/index.jsp

However, we are always looking for others, so do not let this list discourage you.

Being a contributor involves:

- Selecting from a validated, screened search (performed by in-house Information Specialists) epidemiologically sound studies for inclusion.

- Documenting your decisions about which studies to include on an inclusion and exclusion form, which we keep on file.

- Writing the text to a highly structured template (about 1500-3000 words), using evidence from the final studies chosen, within 8-10 weeks of receiving the literature search.

- Working with Clinical Evidence editors to ensure that the final text meets epidemiological and style standards.

- Updating the text every 12 months using any new, sound evidence that becomes available. The Clinical Evidence in-house team will conduct the searches for contributors; your task is simply to filter out high quality studies and incorporate them in the existing text.

If you would like to become a contributor for Clinical Evidence or require more information about what this involves please send your contact details and a copy of your CV, clearly stating the clinical area you are interested in, to CECommissioning@bmigroup.com.

\section{Call for peer reviewers}

Clinical Evidence also needs to recruit a number of new peer reviewers specifically with an interest in the clinical areas stated above, and also others related to general practice. Peer reviewers are healthcare professionals or epidemiologists with experience in evidence-based medicine. As a peer reviewer you would be asked for your views on the clinical relevance, validity, and accessibility of specific topics within the journal, and their usefulness to the intended audience (international generalists and healthcare professionals, possibly with limited statistical knowledge). Topics are usually 1500-3000 words in length and we would ask you to review between 2-5 topics per year. The peer review process takes place throughout the year, and out turnaround time for each review is ideally 10-14 days.

If you are interested in becoming a peer reviewer for Clinical Evidence, please complete the peer review questionnaire at www. clinicalevidence.com/ceweb/contribute/peerreviewer.jsp 